Nevada

Environmental

Restoration

Project

\title{
DOE/NV/11718--289
}

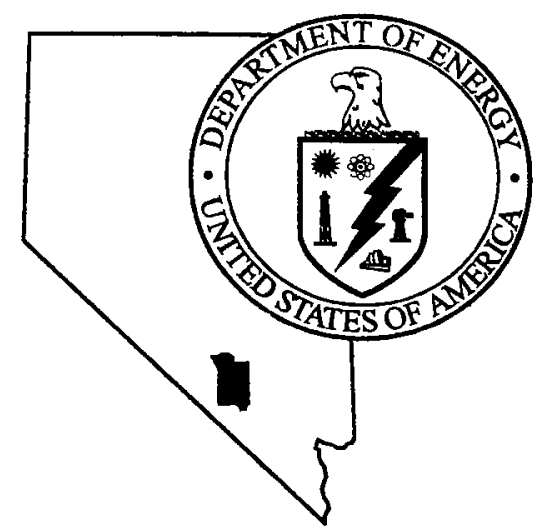

\section{Closure Report \\ For Housekeeping Category \\ Corrective Action Unit 297 \\ Nevada Test Site, Nevada}

Controlled Copy Number:

Revision: 0

March 1999

Distribution A - Approved for public release; further dissemination unlimited.

Environmental Restoration

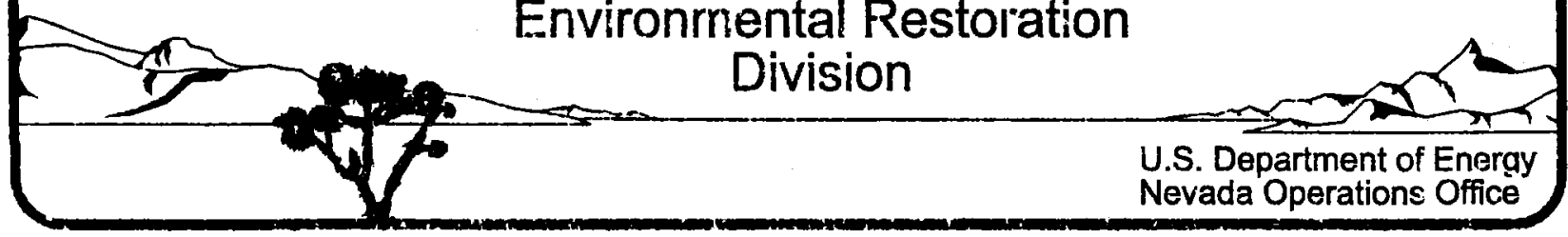


Available to the public from:

U. S. Department of Commerce

National Technical Information Service

5285 Port Royal Road

Springfield, VA 22161

(703) $487-4650$

Available electronically at http://www.doe.gov/bridge. Available to the U.S. Department of Energy and its contractors in paper from:

U.S. Department of Energy

Office of Scientific and Technical Information

P.O. Box 62

Oak Ridge, TN 37831-0062

(423) 576-8401.

Reference herein to any specific commercial product, process, or service by trade name, trademark, manufacturer, or otherwise, does not necessarily constitute or imply its endorsement, recommendation, or favoring by the U.S. Government or any agency thereof or its contractors or subcontractors. 


\title{
CLOSURE REPORT FOR HOUSEKEEPING CATEGORY CORRECTIVE ACTION UNIT 297 NEVADA TEST SITE, NEVADA
}

Controlled Copy No.:

Revision: 0

\author{
Prepared for: \\ U. S. Department of Energy \\ Nevada Operations Office \\ Under Contract No. DE-AC08-96NV11718
}

March 1999 
Corrective Action Unit (CAU) 297 is located at the Nevada Test Site Area 25 Engine Maintenance and Disassembly Building (Building 3900). The CAU consists of one Corrective Action Site (CAS) 25-25-01 which includes two 38-liter (10-gallon) metal containers used in a closed vacuum pump oil recovery system for recirculating oil.

Closure for this site was completed on December 1, 1998 under the guidance of the Housekeeping Category Work Plan (Department of Energy, Nevada Operations Office, 1996) by completing the following activities:

- Two 38-liter (10-gallon) metal containers were removed.

- Associated piping was cut, removed, and sealed.

- Soil containing petroleum hydrocarbons were excavated to a maximum depth of approximately 46 centimeters (18 inches).

- The tanks, associated piping, and impacted soil were disposed of in the Area 6 Hydrocarbon Landfill on January 14, 1999.

Completing the closure activities at the site identified a hydrocarbon release associated with historical operations of the oil recovery system. The petroleum impacted soils are located in a significant power/utility corridor feeding into the building. The first of the underground utilities were identified at a depth of approximately 1 meter ( 3 feet) below ground surface. Due to the extent of the impacted area, confining work space limitations, proximity to utility lines, and limitations to the amount of excavated material for housekeeping closure activities, excavation activities were discontinued. Clean soil was used to backfill over the excavated area.

Following the closure requirements of the Housekeeping Work Plan, only one verification soil sample was collected below the removed containers and piping (EMAD-VPOR-1). Additional samples were collected for quality control (EMAD-VPOR-2) and waste disposition purposes. Samples were analyzed for total petroleum hydrocarbons, Toxicity Characteristic Leaching Procedure (TCLP) volatile organic compounds, TCLP semi-volatile organic compounds, TCLP metals, polychlorinated biphenyls, and organochlorine pesticides.

The soil sample collected below the oil recovery containers and piping contained petroleum hydrocarbons (as oil) at a concentration of 5,200 milligrams per kilogram (mg/kg). This concentration exceeded the Nevada Administrative Code (NAC) Action Level of $100 \mathrm{mg} / \mathrm{kg}$ (NAC 445A.2272). A Spill Notification Report was completed on January 19, 1999 and Nevada Division of Emergency Management Number 99-0120-3281 was assigned. 
Polychlorinated biphenyls (Arochlor 1254) were also detected in the excavated waste soils at a maximum concentration of $7.2 \mathrm{mg} / \mathrm{kg}$, however, the concentrations are below the U.S. Environmental Protection Agency (EPA) Preliminary Remediation Goal concentration for industrial sites of $18 \mathrm{mg} / \mathrm{kg}$ (EPA, 1996).

No other constituents were detected above the analytical method detection limit. The analytical results and test methods are summarized on Table 1 and are detailed in the analytical data package. The table and data package are provided in Appendix A for reference and for use in supporting future characterization and/or closure activities.

Due to the extent of the impacted soil and the work area considerations, the spill associated with this site will be assigned as CAS 25-25-17 under CAU 398. The site will be tracked within Appendix II of the Federal Facility Agreement and Consent Order until corrective actions can be completed at a future date. 


\section{FFACO CORRECTIVE ACTION STTE HOUSEKEEPNG CLOSURE VERIFICATION FORM}

Closure Verifcation Date: Jan. 14. 1999

CAS Number: $25-25-01$

CAUkamber:297

Genera Lowion: Area 25 Engine Maintenance

Elevation: 1071.6 meters

Assembly \& Disassembly (EMAD)

Latude: 36.8062772

(above mean sea level)

Northing .622819 .5

Longitude: 116.3046654

Easting: 532300.7

Coordinate Elevation Data Obtained fron TT. G.P.S. Survey Conducted on lune 23, 1993.

Site Access Route: Hoced southwest on Mercury Mghway to Jackass Fats Road. Tum right (northwest)on Jackass Flats Road (which wums into Road C at the Area 25 Guard Gate [Guard Station 500 ] and proceed to Road H. Tum left (west) on Road H and proced to EMAD Road. Tum left (south) on EMAD Road and proced to the EMAD Facility. The system is mounted on the southeast side of the buiding.

\begin{tabular}{|l|c|}
\hline \multicolumn{1}{|c|}{ Waste lem(s) originaly at Site } & Apparent Waste ype* \\
\hline $\begin{array}{l}\text { Two lo-gallon metal containers, associated } \\
\text { piping, metal structure, and associated } \\
\text { staned sol. }\end{array}$ & $\begin{array}{l}\text { Other: four drums and four luggers } \\
\text { of hydrocarbon-impacted soil, two } \\
\text { metal containers, and associated piping. }\end{array}$ \\
\hline
\end{tabular}

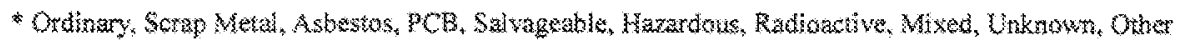

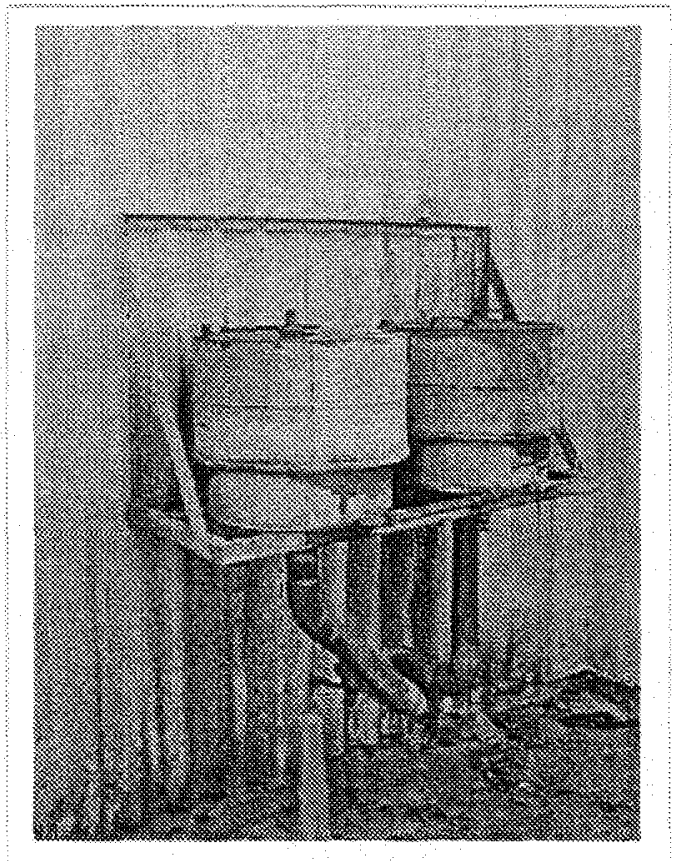

CAS Status Prion To Cleanup Oct. 19,998

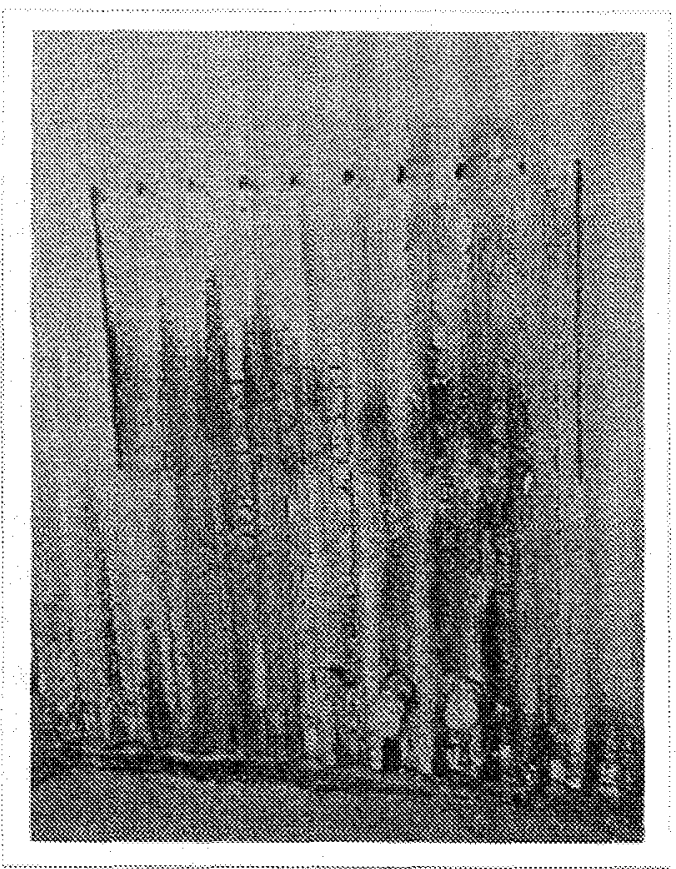

CAS Status After Cleanup

Dec. 2,1998 


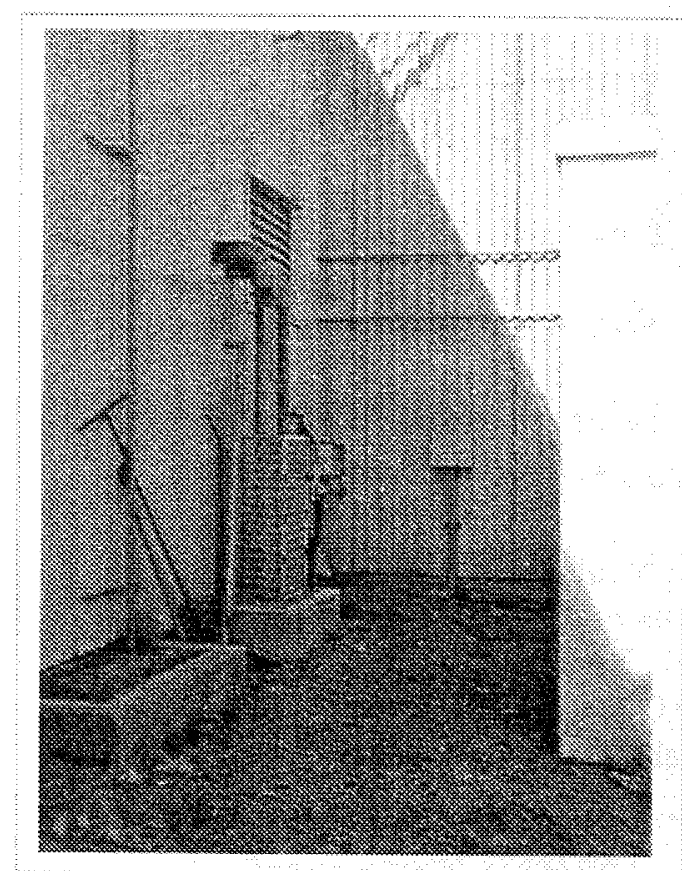

CAS Stus pror To Cleanup Oct. 19.1998

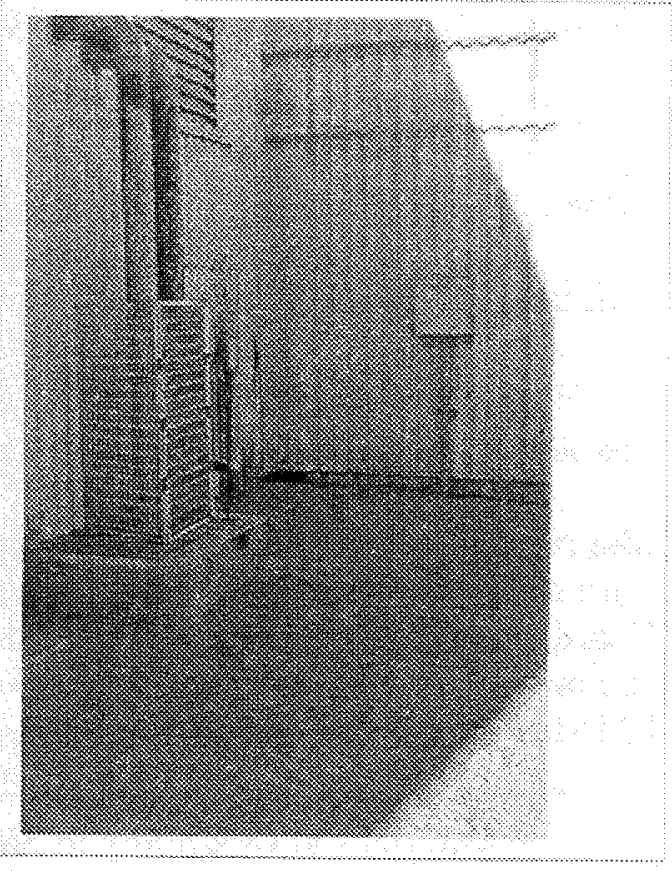

CAS Status Ater Cleanup Dec. $3 \times 1998$

Current Site Description/Observations: The two 10-gallon metal containers, associated piping, and support structure have been removed. The debris and excavated soil were disposed of in the Area 6 Hydrocarbon landfill on Jan. 14, 1999.

\section{No Further Action Required at Corrective Action Site}

Jerry F. Bonn
Corrective Action Coordinator/Designee




\section{REFERENCES}

Federal Facility Agreement and Consent Order, 1996, Agreed to by the Nevada Division of Environmental Protection, the U.S. Department of Energy, and the U.S. Department of Defense.

Nevada Administrative Code, 1998, NAC 445A.2272, "Contamination of Soil: Establishment of Action Levels." Carson City, NV.

U.S. Department Of Energy, Nevada Operations Office, 1996, Housekeeping Category Corrective Action Unit Work Plan, DOE/NV-452. Las Vegas, NV.

U.S. Environmental Protection Agency, 1996, Region 9 Preliminary Remediation Goals, San Francisco, CA. 


\section{APPENDIX A}

\section{ANALYTICAL TEST RESULTS}


TABLE 1 - SUMMARY OF ANALYTICAL RESULTS FOR CAU 297: EMAD VACUUM PUMP OIL RECOVERY

\begin{tabular}{|c|c|c|c|c|c|c|c|c|}
\hline SAMPLE ID \& TYPE & $\underset{(\mathrm{mg} / \mathrm{kg})}{\mathrm{TPH}}$ & $\begin{array}{l}\text { TCLP } \\
\text { VOCs }^{B} \\
(\mathbf{m g} / L)\end{array}$ & $\begin{array}{l}\text { TCLP } \\
\text { SEMI- } \\
\text { VOCs c } \\
\text { (mg/L) }\end{array}$ & $\begin{array}{c}\text { TCLP } \\
\text { METALS } \\
\text { (mg/kg) }\end{array}$ & $\begin{array}{c}\text { PCBs E } \\
(\mathrm{mg} / \mathrm{kg})\end{array}$ & $\begin{array}{c}\text { ORGANO- } \\
\text { CHLORINE } \\
\text { PESTICIDES F } \\
\text { (mg/kg) }\end{array}$ & \multicolumn{2}{|c|}{$\begin{array}{c}\text { GAMMA } \\
\text { (pCi/g) }\end{array}$} \\
\hline $\begin{array}{l}\text { EMAD-VPOR-1 } \\
\text { VERIFICATION }\end{array}$ & 5,200 & ND & ND & ND & ND & ND & \multicolumn{2}{|c|}{ NA } \\
\hline $\begin{array}{l}\text { EMAD-VPOR-2 } \\
\text { DUPLICATE OF } \\
\text { EMAD-VPOR-1 }\end{array}$ & 1,700 & ND & ND & ND & ND & ND & \multicolumn{2}{|c|}{ NA } \\
\hline $\begin{array}{l}\text { EMAD-VPOR-3 } \\
\text { WASTE } \\
\text { CHARACTERIZATION } \\
\text { (DRUMS) } \\
\end{array}$ & 4,700 & ND & ND & ND & $\begin{array}{c}0.7 \\
\text { Arochlor-1254 }\end{array}$ & ND & $\begin{array}{l}\text { Cs-137 } \\
\text { K-40 } \\
\text { Ra-226 } \\
\text { Th-228 }\end{array}$ & $\begin{array}{l}0.165 \\
24.9 \\
1.2 \\
2.28\end{array}$ \\
\hline $\begin{array}{l}\text { EMAD-VPOR-4 } \\
\text { WASTE } \\
\text { CHARACTERIZATION } \\
\text { (LUGGERS) }\end{array}$ & 3,700 & ND & ND & ND & $\begin{array}{c}7.2 \\
\text { Arochlor- } 1254\end{array}$ & ND & $\begin{array}{l}\mathrm{K}-40 \\
\mathrm{Ra}-226 \\
\text { Th-228 } \\
\text { Th-232 } \\
\end{array}$ & $\begin{array}{l}19.6 \\
1.01 \\
1.84 \\
1.26 \\
\end{array}$ \\
\hline
\end{tabular}

All samples were collected on Dec. 1, 1998.

A-TPH: Total (extractable) petroleum hydrocarbons analyzed using U.S. EPA Method 8015 Modified.

B-TCLP VOCs: TCLP Volatile organic compounds analyzed using U.S. EPA Method 8260B.

C-TCLP Semi-VOCs: TCLP Semi-Volatile Organic Compounds analyzed using U.S. EPA Method 8270C.

D-TCLP Metals: Metals analyzed using U.S. EPA Method 7470. Extraction by Method 1311.

E-PCBs: Polychlorinated Biphenyls analyzed using U.S. EPA Method 8082.

F-Organochlorine Pesticides analyzed using U.S. EPA Method 8081A.

TCLP: Toxicity Characteristic Leaching Procedure (40 CFR, Part 261.24).

ND: Not detected above laboratory detection limits.

NA: Not analyzed.

( $\mathrm{mg} / \mathrm{L}$ or $\mathrm{mg} / \mathrm{kg}$ or $\mathrm{pCi} / \mathrm{g}$ ): milligrams per Liter (for liquid), milligrams per kilogram (for soil), picocuries per gram (for gamma spectroscopy). 


\section{CLIENT: Bechtel Nevada}

P.O. Box 98521 , M/S NTS273

Las Vegas, NV 89193-8521

ATTN: Ted Redding

PROJECT NAME: V553

PROJECT NUMBER: 17777

NEL ORDER ID: L9812048

Attached are the analytical results for samples in support of the above referenced project.

Samples were received by NEL in good condition, under chain of custody on 12/3/98.

Samples were analyzed as received.

Should you have any questions or comments, please feel free to contact our Client Services department at (702) 657-1010.

\section{Some results have been flagged as follows:}

$\mathrm{J}$ - This concentration should be considered an estimate due laboratory control sample failure.

Jc - This concentration should be considered an estimate. The continuing calibration check standard did not meet $\mathrm{QC}$ requirements for this analyte.

$\mathrm{Jm}$ - This concentration should be considered an estimate due to probable matrix effects.

Some $Q A$ results have been flagged as follows:

J - This concentration should be considered an estimate due laboratory control sample failure.

Jl - The batch MS and/or MSD were outside acceptance limits. The batch LCS was acceptable

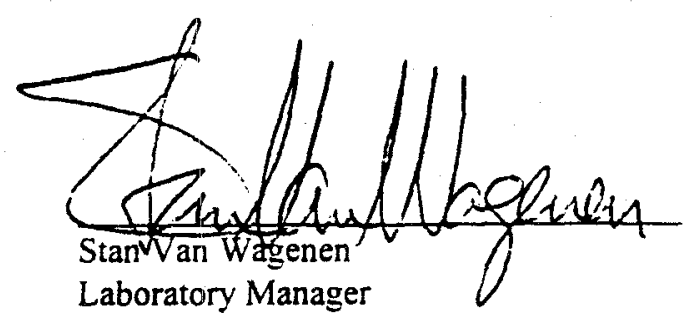

\section{CERTIFICATIONS:}

\begin{tabular}{llll}
\hline & Reno & Las Vegas & S. California \\
\cline { 2 - 4 } Arizona & AZ0520 & AZ0518 & AZ0583 \\
California & 1707 & 2002 & 2264 \\
US Army Corps & Certified & Certified & Certified \\
of Engineers & & &
\end{tabular}

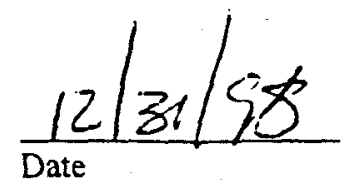

Reno $\frac{\text { Reno }}{\text { Certified }} \quad$ Las Vegas S. Californie Montana Nevada Certified NV052 
NEL LABORATORIES

CLIENT: Bechtel Nevada

PROJECT NAME: V553

PROJECT NUMBER: 17777
CLIENT ID: EMAD-VPOR-1

DATE SAMPLED: $12 / 1 / 98$

NEL SAMPLE ID: L9812048-01

TEST: Organochlorine Pesticides by EPA 8081A, Dec. 1996

MATRIX: Solid

DILUTION: 1

\section{PARAMETER}

Aldrin

alpha-BHC

beta-BHC

delta-BHC

gamma-BHC (Lindane)

Alpha-chlordane

Gamma-chlordane

Chlordane

4. $4^{\circ}-$ DDD

4.4-DDE

4.4.-DDT

Dieldrin

Endosulfan I

Endosulfan II

Endosulfan sulfate

Endrin

Endrin aldehyde

Endrin ketone

Heptachlor

Heptachlor epoxide

Methoxychlor

Toxaphene

QUALITY CONTROL DATA:

Surrogate

Decachlorobiphenyl

Tetrachloro-m-xylene
EXTRACTED: $12 / 8 / 98$

ANALYZED: $12 / 14 / 98$

ANALYST: JRW
Reporting

Limit

5. $\mu \mathrm{g} / \mathrm{kg}$

5. $\mu \mathrm{g} / \mathrm{kg}$

5. $\mu \mathrm{g} / \mathrm{kg}$

5. $\mu \mathrm{g} / \mathrm{kg}$

5. $\mu \mathrm{g} / \mathrm{kg}$

5. $\mu \mathrm{g} / \mathrm{kg}$

5. $\mu \mathrm{g} / \mathrm{kg}$

20. $\mu \mathrm{g} / \mathrm{Kg}$

5. $\mu \mathrm{g} / \mathrm{kg}$

5. $\mu \mathrm{g} / \mathrm{kg}$

5. $\mu \mathrm{g} / \mathrm{kg}$

5. $\mu \mathrm{g} / \mathrm{kg}$

5. $\mu \mathrm{g} / \mathrm{kg}$

5. $\mu \mathrm{g} / \mathrm{kg}$

5. $\mu \mathrm{g} / \mathrm{kg}$

5. $\mu g / \mathrm{kg}$

5. $\mu \mathrm{g} / \mathrm{kg}$

5. $\mu g / \mathrm{kg}$

5. $\mu \mathrm{g} / \mathrm{kg}$

5. $\mu \mathrm{g} / \mathrm{kg}$

20. $\mu \mathrm{g} / \mathrm{kg}$

60. $\mu \mathrm{g} / \mathrm{kg}$
Acceptable Range

$70-130$

$70-130$

ND - Not Detected

This report shall not be reproduced except in full, without the written approval of the laboratory. 
CLIENT: Bechtel Nevada

PROJECT NAME: V553

PROJECT NUMBER: 17777
CLIENT ID: EMAD-VPOR-2

DATE SAMPLED: $12 / 1 / 98$

NEL SAMPLE ID: L9812048-02

TEST: Organochlorine Pesticides by EPA 8081A. Dec. 1996

MATRIX: Solid

EXTRACTED: $12 / 8 / 98$

DILUTION: 1

ANALYZED: $12 / 14 / 98$

ANALYST: JRW

\section{PARAMETER}

Aldrin

alpha-BHC

beta-BHC

delta-BHC

gamma-BHC (Lindane)

Alpha-chlordane

Gamma-chlordane

Chlordane

4. $4^{\circ} \cdot \mathrm{DDD}$

4. $4^{\circ}-\mathrm{DDE}$

4. $4^{\prime}$-DDT

Dieldrin

Endosulfan I

Endosulfan II

Endosulfan sulfate

Endrin

Endrin aldehyde

Endrin ketone

Heptachlor

Heptachlor epoxide

Methoxychlor

Toxaphene

QUALITY CONTROL DATA:

Surrogate

Decachiorobiphenyl

Tetrachloro-m-xylene

\begin{tabular}{l} 
Result \\
\hline ND \\
ND \\
ND \\
ND \\
ND \\
ND \\
ND \\
ND \\
ND Jc \\
ND J \\
ND Jc \\
ND \\
ND \\
ND Jc \\
ND \\
ND Jc \\
ND \\
ND \\
ND Jc \\
ND \\
ND Jc \\
ND
\end{tabular}

Reporting

Limit

5. $\mu \mathrm{g} / \mathrm{kg}$

5. $\mu \mathrm{g} / \mathrm{kg}$

5. $\mu \mathrm{g} / \mathrm{kg}$

5. $\mu \mathrm{g} / \mathrm{kg}$

5. $\mu \mathrm{g} / \mathrm{kg}$

5. $\mu g / k g$

5. $\mu \mathrm{g} / \mathrm{kg}$

20. $\mu g / \mathrm{hg}$

5. $\mu \mathrm{g} / \mathrm{kg}$

5. $\mu \mathrm{g} / \mathrm{kg}$

5. $\mu \mathrm{g} / \mathrm{kg}$

5. $\mu \mathrm{g} / \mathrm{kg}$

5. $\mu \mathrm{\omega} / \mathrm{kg}$

5. $\mu g i k g$

5. $\mu g i k g$

5. $\mu \mathrm{gikg}$

5. $\mu \mathrm{gkg}$

5. $\mu \mathrm{g} k \mathrm{~g}$

5. $\mu \mathrm{g} / \mathrm{kg}$

5. $\mu \mathrm{g} / \mathrm{kg}$

20. $\mu \mathrm{g} / \mathrm{kg}$

60. $\mu \mathrm{gikg}$

ND

\% Recovery

137

113
Acceptable Range

$70-130$

$70-130$

ND - Not Detected

This report shall not be reproduced except in full, without the written approval of the laboratory. 
CLIENT: Bechtel Nevada

PROJECT NAME: V553

PROJECT NUMBER: 17777
CLIENT ID: EMAD-VPOR-3

DATE SAMPLED: $12 / 1 / 98$

NEL SAMPLE ID: L9812048-03

TEST: Organochlorine Pesticides by EPA 8081A, Dec. 1996

MATRIX: Solid

DILUTION: 1
EXTRACTED: $12 / 8 / 98$

ANALYZED: $12 / 14 / 98$

Result

PARAMETER

Aldrin

alpha-BHC

beta-BHC

delta-BHC

gamma-BHC (Lindane)

Alpha-chlordane

Gamma-chlordane

Chlordane

4. $4^{\circ}$-DDD

4. $4^{\circ} \cdot \mathrm{DDE}$

$4.4^{\circ}$-DDT

Dieldrin

Endosulfan I

Endosulfan II

Endosulfan sulfate

Endrin

Endrin aldehyde

Endrin ketone

Heptachlor

Heptachlor epoxide

Methoxychlor

Toxaphene

OUALITY CONTROL DATA:

Surrogate

Decachlorobiphenyl

Tetrachloro-m-xylene
ND

ND

ND

ND

ND

ND

ND

ND

ND Jc

ND J

ND Jc

ND

ND

ND Jc

$\mathrm{ND}$

ND Jc

ND

ND

ND Jc

ND

ND Jc

ND
ANALYST: JRW

Reporting

Limit

5. $\mu g k g$

5. $\mu \mathrm{g} / \mathrm{kg}$

5. $\mu \mathrm{g} / \mathrm{kg}$

5. $\mu \mathrm{g} / \mathrm{kg}$

5. $\mu \mathrm{g} / \mathrm{kg}$

5. $\mu \mathrm{g} / \mathrm{kg}$

5. $\mu \mathrm{g} / \mathrm{kg}$

20. $\mu \mathrm{g} / \mathrm{hg}$

5. $\mu \mathrm{g} / \mathrm{kg}$

5. $\mu g k g$

5. $\mu \mathrm{g} k \mathrm{~g}$

$5 \mu \mathrm{g} / \mathrm{kg}$

5. $\mu \cong \mathrm{kg}$

5. $\mu \mathrm{g} / \mathrm{kg}$

5. $\mu \mathrm{g} / \mathrm{kg}$

5. $\mu \mathrm{g} \mathrm{kg}$

5. $\mu \mathrm{g}-\mathrm{k}$

s. $\mu \mathrm{g} k \mathrm{~g}$

5. $\mu \mathrm{g} k \mathrm{~kg}$

5. $\mu \mathrm{g} / \mathrm{kg}$

20. $\mu \mathrm{kg}$

60. $\mu \mathrm{g} / \mathrm{kg}$

Accentable Range

$70-130$

$70-130$

ND - Not Detected

This report shall not be reproduced except in full, without the written approval of the laboratory. 


$\begin{array}{ll}\text { CLIENT: Bechtel Nevada } & \text { CLIENT ID: EMAD-VPOR-4 } \\ \text { PROJECT NAME: } \quad \text { V553 } & \text { DATE SAMPLED: } 12 / 1 / 98 \\ \text { PROJECT NUMBER: } 17777 & \text { NEL SAMPLE ID: } \quad \text { L9812048-04 }\end{array}$

TEST: Organochlorine Pesticides by EPA 8081A, Dec. 1996

MATRIX: Solid

EXTRACTED: $12 / 8 / 98$

DILUTION: 1

ANALYZED: $12 / 14 / 98$

ANALYST: JRW

\begin{tabular}{|c|c|c|}
\hline PARAMETER & Result & $\begin{array}{l}\text { Reporting } \\
\text { Limit }\end{array}$ \\
\hline Aldrin & ND & 5. $\mu \mathrm{g} / \mathrm{kg}$ \\
\hline alpha-BHC & ND & 5. $\mu \mathrm{g} / \mathrm{kg}$ \\
\hline beta-BHC & ND & 5. $\mu \mathrm{g} / \mathrm{kg}$ \\
\hline delta-BHC & ND & 5. $\mu \mathrm{g} / \mathrm{kg}$ \\
\hline gamma-BHC (Lindane) & ND & 5. $\mu \mathrm{g} / \mathrm{kg}$ \\
\hline Alpha-chlordane & ND & 5. $\mu \mathrm{g} / \mathrm{kg}$ \\
\hline Gamma-chlordane & ND & 5. $\mu \mathrm{g} / \mathrm{kg}$ \\
\hline Chlordane & ND & 20. $\mu \mathrm{g} / \mathrm{Kg}$ \\
\hline $4.4^{\circ}-\mathrm{DDD}$ & ND Jc & 5. $\mu \mathrm{g} / \mathrm{kg}$ \\
\hline $4.4^{\circ}-D D E$ & ND J & 5. $\mu \mathrm{g} / \mathrm{kg}$ \\
\hline $4.4^{\circ} \cdot$ DDT & ND Jc & 5. $\mu \mathrm{g} / \mathrm{kg}$ \\
\hline Dieldrin & ND & 5. $\mu \mathrm{g} / \mathrm{kg}$ \\
\hline Endosulfan I & ND & 5. $\mu \mathrm{g} / \mathrm{kg}$ \\
\hline Endosulfan II & ND Jc & 5. $\mu \mathrm{g} / \mathrm{kg}$ \\
\hline Endosulfan sulfate & ND & 5. $\mu \mathrm{g} / \mathrm{kg}$ \\
\hline Endrin & ND Jc & 5. $\mu \mathrm{g} / \mathrm{kg}$ \\
\hline Endrin aldehyde & ND & 5. $\mu \mathrm{g} / \mathrm{kg}$ \\
\hline Endrin ketone & ND & 5. $\mu \mathrm{g} / \mathrm{kg}$ \\
\hline Heptachlor & ND Jc & 5. $\mu \mathrm{g} / \mathrm{kg}$ \\
\hline Heptachlor epoxide & ND & 5. $\mu \mathrm{g} / \mathrm{kg}$ \\
\hline Methoxychlor & ND Jc & 20. $\mu \mathrm{g} / \mathrm{kg}$ \\
\hline Toxaphene & ND & 60. $\mu \mathrm{g} / \mathrm{kg}$ \\
\hline
\end{tabular}

QUALITY CONTROL DATA:

Surrogate

\% Recovery

Decachlorobiphenyl

Tetrachloro-m-xylene
125

102
Acceptable Range

$70-130$

$70-130$

ND - Not Detected

This report shall not be reproduced except in full, without the written approval of the laboratory. 


$\begin{array}{ll}\text { CLIENT: Bechtel Nevada } & \text { CLIENT ID: EMAD-VPOR-1 } \\ \text { PROJECT NAME: V553 } & \text { DATE SAMPLED: } 12 / 1 / 98 \\ \text { PROJECT NUMBER: } 17777 & \text { NEL SAMPLE ID: } \text { L9812048-01 }\end{array}$

TEST: PCB's (Polychlorinated Biphenyls) by EPA 8082, Dec. 1996 MATRIX: Solid EXTRACTED: 12/8/98 DILUTION: $1 \quad$ ANALYZED: $12 / 9 / 98$ ANALYST: JRW

\begin{tabular}{lcc}
\hline PARAMETER & Result & $\begin{array}{c}\text { Reporting } \\
\text { Limit }\end{array}$ \\
\cline { 2 - 3 } Arochlor-1016 & $\mathrm{ND}$ & $\frac{20 . \mu \mathrm{g} / \mathrm{kg}}{20 . \mu \mathrm{g} / \mathrm{kg}}$ \\
Arochlor-1221 & $\mathrm{ND}$ & $20 . \mu \mathrm{g} / \mathrm{kg}$ \\
Arochlor-1232 & $\mathrm{ND}$ & $20 . \mu \mathrm{g} / \mathrm{kg}$ \\
Arochlor-1242 & $\mathrm{ND}$ & $20 . \mu \mathrm{g} / \mathrm{kg}$ \\
Arochlor-1248 & $\mathrm{ND}$ & $20 . \mu \mathrm{g} / \mathrm{g}$ \\
Arochlor-1254 & $\mathrm{ND}$ & $20 . \mu \mathrm{g} / \mathrm{kg}$ \\
Arochior-1260 & $\mathrm{ND}$ & \\
\hline \hline OUALITY CONTROL DATA: & & Acceptable Range \\
Surrogate & \% Recovery & $70-130$ \\
Decachlorobiphenyl & 205 & $70-130$ \\
Tetrachloro-m-xylene & 100 &
\end{tabular}

ND - Not Detected

This report shall not be reproduced except in full, without the uritten approval of the laboratory. 
CLIENT: Bechtel Nevada

PROJECT NAME: V553

PROJECT NUMBER: 17777
CLIENT ID: EMAD-VPOR-2

DATE SAMPLED: $12 / 1 / 98$

NEL SAMPLE ID: L9812048-02

TEST: PCB's (Polychlorinated Biphenyls) by EPA 8082, Dec. 1996

MATRIX: Solid

EXTRACTED: $12 / 8 / 98$

DILUTION: 1

ANALYZED: $12 / 9 / 98$

ANALYST: JRW

\begin{tabular}{|c|c|c|}
\hline PARAMETER & Result & $\begin{array}{c}\text { Reporting } \\
\text { Limit }\end{array}$ \\
\hline Arochlor-1016 & ND & 20. $\mu \mathrm{g} / \mathrm{kg}$ \\
\hline Arochlor-1221 & ND & 20. $\mu \underline{\mu g} / \mathrm{kg}$ \\
\hline Arochlor- 1232 & ND & 20. $\mu \mathrm{g} / \mathrm{kg}$ \\
\hline Arochlor-1242 & ND & 20). $\mu \mathrm{g} / \mathrm{kg}$ \\
\hline Arochlor-1248 & ND & 20. $\mu g / k g$ \\
\hline Arochior- 1254 & ND & 20. $\mu \mathrm{g} / \mathrm{kg}$ \\
\hline Arochlor- 1260 & ND & 20. $\mu g / \mathrm{kg}$ \\
\hline \multicolumn{3}{|c|}{ QUALITY CONTROL DATA: } \\
\hline Surrogate & $\%$ Recovery & Acceptable Range \\
\hline Decachlorobiphenyl & 70 & $70-130$ \\
\hline Tetrachloro-m-xylene & 78 & $70-130$ \\
\hline
\end{tabular}

ND - Not Detected

This report shall not be reproduced except in full, without the written approval of the laboratory: 
NEL LABORATORIES

CLIENT: Bechtel Nevada

PROJECT NAME: V553

PROJECT NUMBER: 17777
CLIENT ID: EMAD-VPOR-3

DATE SAMPLED: $12 / 1 / 98$

NEL SAMPLE ID: L9812048-03

TEST: PCB's (Polychlorinated Biphenyls) by EPA 8082, Dec. 1996

MATRIX: Solid

DILUTION: 1

\section{PARAMETER}

Arochlor-1016

Arochlor-122!

Arochlor-1232

Arochlor-1242

Arochlor-1248

Arochlor-1254

Arochlor-1260

QUALITY CONTROL DATA:

\section{Surrogate}

Decachlorobiphenyl

Tetrachloro-m-xylene
EXTRACTED: $12 / 8 / 98$

ANALYZED: $12 / 9 / 98$
ANALYST: JRW

\begin{tabular}{l} 
Result \\
\hline $\mathrm{ND}$ \\
$\mathrm{ND}$ \\
$\mathrm{ND}$ \\
$\mathrm{ND}$ \\
$\mathrm{ND}$ \\
$\mathbf{7 0 0} \quad \mu \mathrm{g} / \mathrm{kg}$ \\
$\mathrm{ND}$ \\
\hline
\end{tabular}

\section{$\%$ Recovery}

122

104

\begin{tabular}{l}
$\begin{array}{c}\text { Reporting } \\
\text { Limit }\end{array}$ \\
\hline $20 . \mu \mathrm{g} / \mathrm{kg}$ \\
20. $\mu \mathrm{g} / \mathrm{kg}$ \\
20. $\mu \mathrm{g} / \mathrm{kg}$ \\
20. $\mu \mathrm{g} / \mathrm{kg}$ \\
$20 . \mu \mathrm{g} / \mathrm{kg}$ \\
$20 . \mu \mathrm{g} / \mathrm{kg}$ \\
$20 . \mu \mathrm{g} / \mathrm{kg}$ \\
\hline
\end{tabular}

Acceptable Range

$70-130$

$70-130$

ND - Not Detected

This report shall not be reproduced except in full, without the written approval of the laboratory. 

CLIENT: Bechtel Nevada
PROJECT NAME: V553
CLIENT ID: EMAD-VPOR-4
DATE SAMPLED: $12 / 1 / 98$
PROJECT NUMBER: 17777
NEL SAMPLE ID： L9812048-04

TEST: PCB's (Polychlorinated Biphenyls) by EPA 8082, Dec. 1996

MATRIX: Solid

EXTRACTED: $12 / 8 / 98$

DILUTION: 10

ANALYZED: $12 / 9 / 98$

ANALYST: JRW

\begin{tabular}{|c|c|c|}
\hline PARAMETER & Result & $\begin{array}{c}\text { Reporting } \\
\text { Limit }\end{array}$ \\
\hline Arochlor-1016 & ND & $200 . \mu \mathrm{g} / \mathrm{kg}$ \\
\hline Arochior-1221 & ND & 200. $\mu \cong / \mathrm{kg}$ \\
\hline Arochlor-1232 & ND & 200. $\mu \mathrm{g} / \mathrm{kg}$ \\
\hline Arochlor-1242 & ND & $200 . \mu \mathrm{g} / \mathrm{kg}$ \\
\hline Arochlor-1248 & ND & $200 . \mu \mathrm{g} / \mathrm{kg}$ \\
\hline Arochlor-1254 & $\mu \mathrm{g} / \mathrm{kg}$ & 2(1)0. $\mu \omega / \mathrm{kg}$ \\
\hline Arochlor- 1260 & ND & $200 . \mu \mathrm{g} / \mathrm{kg}$ \\
\hline
\end{tabular}

QUALITY CONTROL DATA:

Surrogate

Decachlorobiphenyl

Tetrachloro-m-xylene

\section{$\%$ Recovery}

110

79

\section{Acceptable Range}

$70-130$

$70-130$

ND - Not Detected

This report shall not be reproduced except in full, without the written approval of the laboratory. 
NEL LABORATORIES

$\begin{array}{llll}\text { CLIENT: } & \text { Bechtel Nevada } & \text { CLIENT ID: } & \text { Method Blank } \\ \text { PROJECT ID: } & \text { V553/17777 } & \text { DATE SAMPLED: } & \text { NA } \\ \text { PROJECT \#: } & 17777 & \text { NEL SAMPLE ID: } & \text { 981208PCBS-BLK }\end{array}$

TEST:

PCB's (Polychlorinated Biphenyls) by EPA 8082, Dec. 1996

METHOD: EPA 8082

MATRIX: Solid

$\begin{array}{ll}\text { ANALYST: } & \text { JRW } \\ \text { EXTRACTED: } & 12 / 8 / 98 \\ \text { ANALYZED: } & 12 / 9 / 98\end{array}$

\section{PARAMETER}

Arochlor-1016

Arochlor-1221

Arochlor-1232

Arochlor-1242

\begin{tabular}{l} 
Result \\
\hline ND \\
ND \\
ND \\
ND \\
ND \\
ND \\
ND
\end{tabular}

$12 / 9 / 98$

Arochlor-1248

Arochlor-1254

Arochlor-1260

\section{$\%$ Recovery}

Surrogate

Decachlorobipheny]

Tetrachloro-m-xylene
122

123
Reporting

20. $\mu \mathrm{g} / \mathrm{kg}$

20. $\mu \mathrm{g} / \mathrm{kg}$

20. $\mu \mathrm{g} / \mathrm{kg}$

20. $\mu \mathrm{g} / \mathrm{kg}$

20. $\mu \mathrm{g} / \mathrm{kg}$

20. $\mu \mathrm{g} / \mathrm{kg}$

20. $\mu \mathrm{g} / \mathrm{kg}$

ND - Not Detected

This report shall not be reproduced except in full, without the uritten approval of the laboratory 


$\begin{array}{llll}\text { CLIENT: } & \text { Bechtel Nevada } & \text { CLIENT ID: } & \text { Method Blank } \\ \text { PROJECT ID: } & \text { V553/17777 } & \text { DATE SAMPLED: } & \text { NA } \\ \text { PROJECT \#: } & 17777 & \text { NEL SAMPLE ID: } & 981208 \text { PSTS-BLK }\end{array}$

TEST:

METHOD:

Organochlorine Pesticides by EPA 8081A, Dec. 1996

MATRIX:

EPA 8081

Solid

$\begin{array}{ll}\text { ANALYST: } & \text { JRW } \\ \text { EXTRACTED: } & 12 / 8 / 98 \\ \text { ANALYZED: } & 12 / 14 / 98\end{array}$

\section{PARAMETER}

Aldrin

alpha-BHC

beta-BHC

delta-BHC

gamma-BHC (Lindane)

Alpha-chlordane

Gamma-chlordane

Chlordane

\section{Result}

ND

4. $4^{\circ}$-DDD

4. $4^{\circ}$-DDE

4.4-DDT

Dieldrin

Endosulfan I

Endosulfan II

Endosulfan sulfate

Endrin

Endrin aldehyde

Endrin ketone

Heptachlor

Heptachlor epoxide

Methoxychlor

Toxaphene

QUALITY CONTROL DATA

\section{Surrogate}

Decachlorobiphenyl

Tetrachloro-m-xylene

$\%$ Recovery

108

87
Reporting

Limit

5. $\mu \mathrm{g} / \mathrm{kg}$

5. $\mu \mathrm{g} / \mathrm{kg}$

5. $\mu \mathrm{g} / \mathrm{kg}$

5. $\mu \mathrm{g} / \mathrm{kg}$

5. $\mu \mathrm{g} / \mathrm{kg}$

5. $\mu \mathrm{g} / \mathrm{kg}$

5. $\mu \mathrm{g} / \mathrm{kg}$

20. $\mu \mathrm{g} / \mathrm{Kg}$

5. $\mu \mathrm{g} / \mathrm{kg}$

5. $\mu \mathrm{g} / \mathrm{kg}$

5. $\mu \mathrm{g} / \mathrm{kg}$

5. $\mu g / \mathrm{kg}$

5. $\mu \mathrm{g} / \mathrm{kg}$

5. $\mu \mathrm{g} / \mathrm{kg}$

5. $\mu \mathrm{g} / \mathrm{kg}$

5. $\mu \mathrm{g} / \mathrm{kg}$

5. $\mu \mathrm{g} / \mathrm{kg}$

5. $\mu \mathrm{g} / \mathrm{kg}$

5. $\mu \mathrm{g} / \mathrm{kg}$

5. $\mu \mathrm{g} / \mathrm{kg}$

20. $\mu \mathrm{g} / \mathrm{kg}$

60. $\mu \mathrm{g} / \mathrm{kg}$

\section{Acceptable Range}

$70-130$

$70-130$

ND - Not Detected

This report shall not be reproduced except in full, without the written approval of the laboratory. 
CLIENT: Bechtel Nevada

PROJECT NAME:

V5.53

PROJECT NUMBER: 17777

CLIENT ID:

DATE SAMPLED: $12 / 1 / 98$

NEL SAMPLE ID: L9812048-0I

TEST:

TCLP-8 Metals

MATRIX: Solid

\begin{tabular}{|c|c|c|c|c|c|c|c|}
\hline PARAMETER & $\begin{array}{c}\text { RESULT } \\
\mathrm{mg} / \mathrm{L} \\
\end{array}$ & $\begin{array}{c}\text { REPORTING } \\
\text { LIMIT } \\
\end{array}$ & D. F. & METHOD & $\begin{array}{c}\text { TCLP/STLC } \\
\text { EXTRACTION } \\
\text { DATE } \\
\end{array}$ & DIGESTED & ANALYZED \\
\hline Arsenic & ND & $0.5 \mathrm{mg} / \mathrm{L}$ & 5 & EPA 6010 & $12 / 8 / 98$ & $12 / 9 / 98$ & $12 / 9 / 98$ \\
\hline Barium & ND & 5. $\mathrm{mg} / \mathrm{L}$ & 5 & EPA 6010 & $12 / 8 / 98$ & $12 / 9 / 98$ & $12 / 9 / 98$ \\
\hline Cadmium & ND & $0.05 \mathrm{mg} / \mathrm{L}$ & 5 & EPA 6010 & $12 / 8 / 98$ & $12 / 9 / 98$ & $12 / 9 / 98$ \\
\hline Chromium & ND & $0.05 \mathrm{mg} / \mathrm{L}$ & 5 & EPA 6010 & $12 / 8 / 98$ & $12 / 9 / 98$ & $12 / 9 / 98$ \\
\hline Lead & ND & $0.25 \mathrm{mg} / \mathrm{L}$ & 5 & EPA 6010 & $12 / 8 / 98$ & $12 / 9 / 98$ & $12 / 9 / 98$ \\
\hline Mercury & ND & $0.002 \mathrm{mg} / \mathrm{L}$ & 10 & EPA $7470 A$ & $12 / 8 / 98$ & $12 / 9 / 98$ & $12 / 9 / 98$ \\
\hline Selenium & ND & $0.5 \mathrm{mg} / \mathrm{L}$ & 5 & EPA 6010 & $12 / 8 / 98$ & $12 / 9 / 98$ & $12 / 9 / 98$ \\
\hline Silver & ND & $0.1 \mathrm{mg} / \mathrm{L}$ & 5 & EPA 6010 & $12 / 8 / 98$ & $12 / 9 / 98$ & $12 / 9 / 98$ \\
\hline
\end{tabular}

D.F. - Dilution Factor

ND - Not Detected

This report shall not be reproduced except in full, without the written approval of the laboratory. 
CLIENT: Bechtel Nevada
PROJECT NAME: V553
PROJECT NUMBER: 17777

TEST: $\quad$ TCLP-8 Metals

MATRIX: Solid
CLIENT ID:

DATE SAMPLED: $12 / 1 / 98$

NEL SAMPLE ID: L9812048-02

\begin{tabular}{|c|c|c|c|c|c|c|c|}
\hline PARAMETER & $\begin{array}{c}\text { RESULT } \\
\mathrm{mg} / \mathrm{L} \\
\end{array}$ & $\begin{array}{c}\text { REPORTING } \\
\text { LIMIT } \\
\end{array}$ & D. F. & METHOD & $\begin{array}{c}\text { TCLP/STLC } \\
\text { EXTRACTION } \\
\text { DATE } \\
\end{array}$ & DIGESTED & ANALYZED \\
\hline Arsenic & ND & $0.5 \mathrm{mg} / \mathrm{L}$ & 5 & EPA 6010 & $12 / 8 / 98$ & $12 / 9 / 98$ & $12 / 9 / 98$ \\
\hline Bariurn & ND & $5 . \mathrm{mg} / \mathrm{L}$ & 5 & EPA 6010 & $12 / 8 / 98$ & $12 / 9 / 98$ & $12 / 9 / 98$ \\
\hline Cadmium & ND & $0.05 \mathrm{mg} / \mathrm{L}$ & 5 & EPA 6010 & $.12 / 8 / 98$ & 12/9/98 & 12/9/98 \\
\hline Chromium & ND & $0.05 \mathrm{mg} / \mathrm{L}$ & 5 & EPA 6010 & $12 / 8 / 98$ & 12/9/98 & $12 / 9 / 98$ \\
\hline Lead & ND & $0.25 \mathrm{mg} / \mathrm{L}$ & 5 & EPA 6010 & $12 / 8 / 98$ & $12 / 9 / 98$ & $12 / 9 / 98$ \\
\hline Mercury & ND & $0.002 \mathrm{mg} / \mathrm{L}$ & 10 & EPA $7470 \mathrm{~A}$ & $12 / 8 / 98$ & $12 / 9 / 98$ & $12 / 9 / 98$ \\
\hline Selenium & ND & $0.5 \mathrm{mg} / \mathrm{L}$ & 5 & EPA 6010 & $12 / 8 / 98$ & $12 / 9 / 98$ & $12 / 9 / 98$ \\
\hline Silver & ND & $0.1 \mathrm{mg} / \mathrm{L}$ & 5 & EPA 6010 & $12 / 8 / 98$ & $12 / 9 / 98$ & $12 / 9 / 98$ \\
\hline
\end{tabular}

D.F. - Dilution Factor

ND - Not Detected

This report shall not be reproduced except in full, without the written approval of the laboratory. 
CLIENT: Bechtel Nevada

PROJECT NAME: V553

PROJECT NUMBER: 17777
CLIENT ID:

DATE SAMPLED: $12 / 1 / 98$

NEL SAMPLE ID: L9812048-03

TEST:

TCLP-8 Metals

MATRIX: $\quad$ Solid

\begin{tabular}{|c|c|c|c|c|c|c|c|}
\hline PARAMETER & $\begin{array}{c}\text { RESULT } \\
\mathrm{mg} / \mathrm{L} \\
\end{array}$ & $\begin{array}{c}\text { REPORTING } \\
\text { LIMIT } \\
\end{array}$ & D. $F$. & METHOD & $\begin{array}{c}\text { TCLP/STLC } \\
\text { EXTRACTION } \\
\text { DATE } \\
\end{array}$ & DIGESTED & ANALYZED \\
\hline Arsenic & ND & $0.5 \mathrm{mg} / \mathrm{L}$ & 5 & EPA 6010 & $12 / 8 / 98$ & $12 / 9 / 98$ & $12 / 9 / 98$ \\
\hline Barium & ND & 5. $\mathrm{mg} / \mathrm{L}$ & 5 & EPA 6010 & $12 / 8 / 98$ & $12 / 9 / 98$ & $12 / 9 / 98$ \\
\hline Cadmium & ND & $0.05 \mathrm{mg} / \mathrm{L}$ & 5 & EPA 6010 & $12 / 8 / 98$ & $12 / 9 / 98$ & $12 / 9 / 98$ \\
\hline Chromium & ND & $0.05 \mathrm{mg} / \mathrm{L}$ & 5 & EPA 6010 & $12 / 8 / 98$ & $12 / 9 / 98$ & $12 / 9 / 98$ \\
\hline Lead & ND & $0.25 \mathrm{mg} / \mathrm{L}$ & 5 & EPA 6010 & $12 / 8 / 98$ & $12 / 9 / 98$ & $12 / 9 / 98$ \\
\hline Mercury & ND & $0.002 \mathrm{mg} / \mathrm{L}$ & 10 & EPA 7470A & $12 / 8 / 98$ & $12 / 9 / 98$ & $12 / 9 / 98$ \\
\hline Selenium & ND & $0.5 \mathrm{mg} / \mathrm{L}$ & 5 & EPA 6010 & $12 / 8 / 98$ & $12 / 9 / 98$ & $12 / 9 / 98$ \\
\hline Silver & ND & $0.1 \mathrm{mg} / \mathrm{L}$ & 5 & EPA 6010 & $12 / 8 / 98$ & $12 / 9 / 98$ & $12 / 9 / 98$ \\
\hline
\end{tabular}

D.F. - Dilution Factor

ND - Not Detected

This report shall not be reproduced except in full, without the written approval of the laboratory. 
CLIENT: Bechtel Nevada

PROJECT NAME: V 553

PROJECT NUMBER: 17777
CLIENT ID:

DATE SAMPLED: 12/1/98

NEL SAMPLE ID: L9812048-04

TEST:

TCLP-8 Metals

MATRIX: $\quad$ Solid

\begin{tabular}{|c|c|c|c|c|c|c|c|}
\hline PARAMETER & $\begin{array}{c}\text { RESULT } \\
\mathrm{mg} / \mathrm{L} \\
\end{array}$ & $\begin{array}{c}\text { REPORTING } \\
\text { LIMIT } \\
\end{array}$ & D. F. & METHOD & $\begin{array}{c}\text { TCLP/STLC } \\
\text { EXTRACTION } \\
\text { DATE } \\
\end{array}$ & DIGESTED & ANALYZED \\
\hline Arsenic & ND & $0.5 \mathrm{mg} / \mathrm{L}$ & 5 & EPA 6010 & $12 / 8 / 98$ & $12 / 9 / 98$ & $12 / 9 / 98$ \\
\hline Barium & ND & $5 . \mathrm{mg} / \mathrm{L}$ & 5 & EPA 6010 & $12 / 8 / 98$ & $12 / 9 / 98$ & $12 / 9 / 98$ \\
\hline Cadmium & ND & $0.05 \mathrm{mg} / \mathrm{L}$ & 5 & EPA 6010 & $12 / 8 / 98$ & $12 / 9 / 98$ & $12 / 9 / 98$ \\
\hline Chromium & ND & $0.05 \mathrm{mg} / \mathrm{L}$ & 5 & EPA 6010 & $12 / 8 / 98$ & $12 / 9 / 98$ & $12 / 9 / 98$ \\
\hline Lead & ND & $0.25 \mathrm{mg} / \mathrm{L}$ & 5 & EPA 6010 & $12 / 8 / 98$ & $12 / 9 / 98$ & $12 / 9 / 98$ \\
\hline Mercury & ND & $0.002 \mathrm{mg} / \mathrm{L}$ & 10 & EPA $7470 A$ & $12 / 8 / 98$ & $12 / 9 / 98$ & $12 / 9 / 98$ \\
\hline Selenium & ND & $0.5 \mathrm{mg} / \mathrm{L}$ & 5 & EPA 6010 & $12 / 8 / 98$ & $12 / 9 / 98$ & $12 / 9 / 98$ \\
\hline Silver & ND & $0.1 \mathrm{mg} / \mathrm{L}$ & 5 & EPA 6010 & $12 / 8 / 98$ & $12 / 9 / 98$ & $12 / 9 / 98$ \\
\hline
\end{tabular}

D.F. - Dilution Factor

ND - Not Detected

This report shall not be reproduced except in full, without the written approval of the laboratory. 
NEL LABORATORIES

$\begin{array}{llll}\text { CLIENT: } & \text { Bechtel Nevada } & \text { CLIENT ID: } & \text { Method Blank } \\ \text { PROJECT ID: } & \text { V553/17777 } & \text { DATE SAMPLED: } & \text { NA } \\ \text { PROJECT \#: } & 17777 & \text { NEL SAMPLE ID: } & \text { L12048-HG02-BLK }\end{array}$

TEST: $\quad$ TCLP by EPA 1311, July 1992 \& Mercury by EPA 7470A, July 1992

MATRIX: TCLP Extract

\begin{tabular}{|c|c|c|c|c|c|c|c|}
\hline \multirow[b]{2}{*}{ PARAMETER } & \multirow[b]{2}{*}{ RESULT } & \multirow{2}{*}{$\begin{array}{l}\text { REPORTING } \\
\text { LIMIT }\end{array}$} & \multicolumn{4}{|c|}{$\begin{array}{l}\text { TCLP/STLC } \\
\text { EXTRACTION }\end{array}$} & \\
\hline & & & D. F. & METHOD & DATE & DIGESTED & ANALYZED \\
\hline Mercury & ND & $0.002 \mathrm{mg} / \mathrm{L}$ & 10 & EPA $7470 A$ & $12 / 8 / 98$ & $12 / 9 / 98$ & $12 / 9 / 98$ \\
\hline
\end{tabular}

D.F. - Dilution Factor

ND - Not Detected

This report shall not be reproduced except in full, without the written approval of the laboraton: 


\begin{tabular}{|c|c|c|c|c|c|c|c|}
\hline $\begin{array}{l}\text { CLIENT: } \\
\text { PROJECT ID: } \\
\text { PROJECT \#: }\end{array}$ & $\begin{array}{l}\text { Bechtel Nevada } \\
\text { V553/17777 } \\
17777\end{array}$ & & & $\begin{array}{l}\text { CLIENT ID } \\
\text { DATE SAM } \\
\text { NEL SAMP }\end{array}$ & $\begin{array}{ll} & \text { Metl } \\
\text { ED: } & \text { NA } \\
\text { ID: } & \text { L12C }\end{array}$ & $\begin{array}{l}\text { d Blank } \\
\text { 3i-BLK }\end{array}$ & \\
\hline \multirow[t]{2}{*}{$\begin{array}{l}\text { TEST: } \\
\text { MATRIX: }\end{array}$} & \multicolumn{7}{|c|}{$\begin{array}{l}\text { TCLP by EPA 1311, July } 1992 \& 7 \text { Metals by EPA } 6010 A \text {, July } 1992 \\
\text { TCLP Extract }\end{array}$} \\
\hline & & REPORTING & & & $\begin{array}{l}\text { CLP/STL } \\
\text { TRACTIC }\end{array}$ & & \\
\hline PARAMETER & RESULT & LIMIT & $\underline{\text { D. F. }}$ & METHOD & DATE & DIGESTED & ANALYZED \\
\hline Arsenic & ND & $0.5 \mathrm{mg} / \mathrm{L}$ & 5 & EPA 6010 & $12 / 8 / 98$ & $12 / 9 / 98$ & $12 / 9 / 98$ \\
\hline Barium & ND & $5 \mathrm{mg} / \mathrm{L}$ & 5 & EPA 6010 & $12 / 8 / 98$ & $12 / 9 / 98$ & $12 / 9 / 98$ \\
\hline Cadmium & ND & $0.05 \mathrm{mg} / \mathrm{L}$ & 5 & EPA 6010 & $12 / 8 / 98$ & $12 / 9 / 98$ & $12 / 9 / 98$ \\
\hline Chromium & ND & $0.05 \mathrm{mg} / \mathrm{L}$ & 5 & EPA 6010 & $12 / 8 / 98$ & $12 / 9 / 98$ & $12 / 9 / 98$ \\
\hline Lead & ND & $0.25 \mathrm{mg} / \mathrm{L}$ & 5 & EPA 6010 & $12 / 8 / 98$ & $12 / 9 / 98^{\circ}$ & $12 / 9 / 98$ \\
\hline Selenium & ND & $0.5 \mathrm{mg} / \mathrm{L}$ & 5 & EPA 6010 & $12 / 8 / 98$ & $12 / 9 / 98$ & $12 / 9 / 98$ \\
\hline Silver & ND & $0.1 \mathrm{mg} / \mathrm{L}$ & 5 & EPA 6010 & $12 / 8 / 98$ & $12 / 9 / 98$ & $12 / 9 / 98$ \\
\hline
\end{tabular}

D.F. - Dilution Factor

ND - Not Detected

This report shall not be reproduced except in full, without the written approval of the laboratory. 


\section{NEL LABORATORIES}

$\begin{array}{llll}\text { CLIENT: } & \text { Bechtel Nevada } & \text { CLIENT ID: } & \text { EMAD-VPOR-1 } \\ \text { PROJECT ID: } & \text { V553/17777 } & \text { DATE SAMPLED: } & 12 / 1 / 98 \\ \text { PROJECT \#: } & 17777 & \text { NEL SAMPLE ID: } & \text { L9812048-01 }\end{array}$

TEST:

TCLP by EPA 1311, July 1992 \& Volatile Organics by EPA 8260B, Dec. 1996

METHOD: $\quad$ EPA 8260A

MATRIX: Solid

DILUTION: 1

TCLP EXTRACT DATE: $12 / 9 / 98$

EXTRACTED: $\quad 12 / 11 / 98$

ANALYZED: $\quad 12 / 11 / 98$

\begin{tabular}{lcc}
\hline PARAMETER & $\begin{array}{c}\text { Result } \\
\mathbf{m g} / \mathrm{L}\end{array}$ & $\begin{array}{c}\text { Reporting } \\
\text { Limit }\end{array}$ \\
\cline { 2 - 3 } & $\mathrm{ND}$ & $0.1 \mathrm{mg} / \mathrm{L}$ \\
Benzene & $\mathrm{ND}$ & $0.1 \mathrm{mg} / \mathrm{L}$ \\
Carbon tetrachloride & $\mathrm{ND}$ & $0.1 \mathrm{mg} / \mathrm{L}$ \\
Chiorobenzene & $\mathrm{ND}$ & $0.1 \mathrm{mg} / \mathrm{L}$ \\
Chioroform & $\mathrm{ND}$ & $0.1 \mathrm{mg} / \mathrm{L}$ \\
l.t-Dichlorobenzene (p-DCB) & $\mathrm{ND}$ & $0.1 \mathrm{mg} / \mathrm{L}$ \\
1.2-Dichloroethane (1.2-DCA) & $\mathrm{ND}$ & $0.1 \mathrm{mg} / \mathrm{L}$ \\
1.1-Dichloroethene (1,1-DCE) & $\mathrm{ND}$ & $2 . \mathrm{mg} / \mathrm{L}$ \\
Methyl Ethyl Ketone & $\mathrm{ND}$ & $0.1 \mathrm{mg} / \mathrm{L}$ \\
Tetrachioroethene (PCE) & $\mathrm{ND}$ & $0.1 \mathrm{mg} / \mathrm{L}$ \\
Trichloroethene & $\mathrm{ND}$ & $0.1 \mathrm{mg} / \mathrm{L}$ \\
Vinyl chioride & &
\end{tabular}

QUALITY CONTROL DATA:

Surrogate

t-Bromofluorobenzene

Dibromofluoromethane

Toluene-d8
\% Recovery

86

93

99
Acceptable Range

$74-121$

$80-120$

$81-117$

VD - Not Detected

This report shall not be reproduced except in full, without the written approval of the laboratory. 
NEL LABORATORIES

$\begin{array}{llll}\text { CLIENT: } & \text { Bechtel Nevada } & \text { CLIENT ID: } & \text { EMAD-VPOR-1 } \\ \text { PROJECT ID: } & \text { V553/17777 } & \text { DATE SAMPLED: } & \text { 12/1/98 } \\ \text { PROJECT \#: } & 17777 & \text { NEL SAMPLE ID: } & \text { L9812048-01 }\end{array}$

TEST: $\quad$ TCLP by EPA 1311, Juiy 1992 \& Semivolatile Organics by EPA Method 8270C, Dec. 1996

METHOD: $\quad$ EPA 8270

MATRIX: Solid

DILUTION: 1 TCLP EXTRACT DATE: $12 / 8 / 98$

EXTRACTED: $\quad 12 / 10 / 98$

ANALYZED: $\quad 12 / 13 / 98$

\section{PARAMETER}

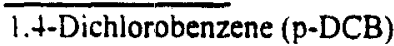

2.t-Dinitrotoluene (DNT)

Hexachlorobenzene

Hexachlorobutadiene

Hexachloroethane

2-Methylphenol

3.t-Methylphenol (isomeric pair)

Nitrobenzene

Pentachlorophenol

Pyridine

2.4.5-Trichlorophenol

2.4.6-Trichlorophenol

\begin{tabular}{c}
$\begin{array}{c}\text { Result } \\
\mathbf{m g} / \mathbf{L}\end{array}$ \\
\hline ND \\
ND \\
ND \\
ND \\
ND \\
ND \\
ND \\
ND \\
ND \\
ND \\
ND \\
ND
\end{tabular}

Result

ND

ND

ND

ND

ND

ND

ND

ND

ND
Reporting .

Limit

$0.1 \mathrm{mg} / \mathrm{L}$

$0.1 \mathrm{mg} / \mathrm{L}$

$0.1 \mathrm{mg} / \mathrm{L}$.

$0.1 \mathrm{mg} / \mathrm{L}$

$0.1 \mathrm{mg} / \mathrm{L}$

$0.1 \mathrm{mg} / \mathrm{L}$

$0.1 \mathrm{mg} / \mathrm{L}$

$0.1 \mathrm{mg} / \mathrm{L}$

$0.1 \mathrm{mg} / \mathrm{L}$

$0.1 \mathrm{mg} / \mathrm{L}$

$0.1 \mathrm{mg} / \mathrm{L}$

$0.1 \mathrm{mg} / \mathrm{L}$

QUALITY CONTROL DATA:

Surrogate

2.4.6-Tribromophenol

2-Fluorobiphenyl

2-Fluorophenol

Vitrobenzene-d5

p-Terphenyl-d 44

Phenol-d5
\% Recoverv

64

67

37

60

- 69

25
Acceptable Range

$10-123$

$43-116$

$21-100$

$35-114$

$33-141$

$10-94$

VD - Not Detected

This report shall not be reproduced except in full, without the written approval of the laboratory. 
NEL LABORATORIES

\begin{tabular}{llll}
\hline CLIENT: & Bechtel Nevada & CLIENT ID: & EMAD-VPOR-2 \\
PROJECT ID: & V $553 / 17777$ & DATE SAMPLED: & $12 / 1 / 98$ \\
PROJECT \#: & 17777 & NEL SAMPLE ID: & L9812048-02
\end{tabular}

TEST: $\quad$ TCLP by EPA 1311, July 1992 \& Volatile Organics by EPA 8260B, Dec. 1996

METHOD: EPA 8260A TCLP EXTRACT DATE: $12 / 9 / 98$

$\begin{array}{llll}\text { MATRIX: } & \text { Solid } & \text { EXTRACTED: } & \text { 12/11/98 }\end{array}$

$\begin{array}{llll}\text { DILUTION: } 1 & \text { ANALYZED: } & 12 / 11 / 98\end{array}$

\begin{tabular}{lcc}
\hline & $\begin{array}{c}\text { Result } \\
\mathbf{m g} / \mathrm{L}\end{array}$ & $\begin{array}{c}\text { Reporting } \\
\text { Limit }\end{array}$ \\
\cline { 2 - 3 } PARAMETER & $\mathrm{ND}$ & $0.1 \mathrm{mg} / \mathrm{L}$ \\
Carbene & $\mathrm{ND}$ & $0.1 \mathrm{mg} / \mathrm{L}$ \\
Chlorobenzene & $\mathrm{ND}$ & $0.1 \mathrm{mg} / \mathrm{L}$ \\
Chloroform & $\mathrm{ND}$ & $0.1 \mathrm{mg} / \mathrm{L}$ \\
1.4-Dichlorobenzene (p-DCB) & $\mathrm{ND}$ & $0.1 \mathrm{mg} / \mathrm{L}$ \\
1.2-Dichloroethane (1,2-DCA) & $\mathrm{ND}$ & $0.1 \mathrm{mg} / \mathrm{L}$ \\
1.1-Dichloroethene (1,1-DCE) & $\mathrm{ND}$ & $0.1 \mathrm{mg} / \mathrm{L}$ \\
Methyl Ethyl Ketone & $\mathrm{ND}$ & $2 . \mathrm{mg} / \mathrm{L}$ \\
Tetrachloroethene (PCE) & ND & $0.1 \mathrm{mg} / \mathrm{L}$ \\
Trichloroethene & ND & $0.1 \mathrm{mg} / \mathrm{L}$ \\
Vinyl chloride & ND & $0.1 \mathrm{mg} / \mathrm{L}$
\end{tabular}

DUALITY CONTROL DATA:

iurrogate

t-Bromofluorobenzene

Dibromofluoromethane

Toluene-d8

\section{$\%$ Recovery}

87

95

99
Acceptable Range

$74-121$

$80-120$

$81-117$

VD - Not Detected

This report shall not be reproduced except in full, without the written approval of the laboratory. 
NEL LABORATORIES

\begin{tabular}{llll}
\hline CLIENT: & Bechtel Nevada & CLIENT ID: & EMAD-VPOR-2 \\
PROJECT ID: & V553/17777 & DATE SAMPLED: & $12 / 1 / 98$ \\
PROJECT \#: & 17777 & NEL SAMPLE ID: & L9812048-02
\end{tabular}

TEST: $\quad$ TCLP by EPA 1311, July 1992 \& Semivolatile Organics by EPA Method 8270C, Dec. 1996

METHOD: $\quad$ EPA 8270

MATRIX: Solid

DILUTION: 1

TCLP EXTRACT DATE: $12 / 8 / 98$

EXTRACTED: $\quad 12 / 10 / 98$

ANALYZED: $\quad 12 / 13 / 98$

\section{PARAMETER}

1.4-Dichlorobenzene (p-DCB)

2.4-Dinitrotoluene (DNT)

Hexachlorobenzene

Hexachlorobutadiene

Hexachloroethane

2-Methylphenol

3.4-Methylphenol (isomeric pair)

Vitrobenzene

Pentachlorophenol

Pyridine

2.4.5-Trichlorophenol

2.4.6-Trichlorophenol

\begin{tabular}{ccc}
$\begin{array}{c}\text { Result } \\
\mathrm{mg} / \mathrm{L}\end{array}$ & $\begin{array}{c}\text { Reporting } \\
\text { Limit }\end{array}$ \\
\cline { 1 - 1 } ND & Jm & $0.1 \mathrm{mg} / \mathrm{L}$ \\
ND & & $0.1 \mathrm{mg} / \mathrm{L}$ \\
ND & $0.1 \mathrm{mg} / \mathrm{L}$ \\
ND & Jm & $0.1 \mathrm{mg} / \mathrm{L}$ \\
ND & Jm & $0.1 \mathrm{mg} / \mathrm{L}$ \\
ND & $0.1 \mathrm{mg} / \mathrm{L}$ \\
ND & $0.1 \mathrm{mg} / \mathrm{L}$ \\
ND & Jm & $0.1 \mathrm{mg} / \mathrm{L}$ \\
ND & $0.1 \mathrm{mg} / \mathrm{L}$ \\
ND Jm & $0.1 \mathrm{mg} / \mathrm{L}$ \\
ND & $0.1 \mathrm{mg} / \mathrm{L}$ \\
ND & $0.1 \mathrm{mg} / \mathrm{L}$
\end{tabular}

UALITY CONTROL DATA:

iurrogate

-4.6-Tribromophenol

-Fluorobiphenyl

-Fluorophenol

vitrobenzene-d5

-Terphenyl-d 14

'henol-d5

\section{$\%$ Recoverv}

60

47

$9 \mathrm{Jm}$

$20 \quad \mathrm{Jm}$

65

13
Accentable Range

$10-123$

$43-116$

21- 100

$35-114$

$33-141$

$10-94$

:D - Not Detected

his report shall not be reproduced except in full, without the written approval of the laboratory. 
NEL LABORATORIES

\begin{tabular}{llll}
\hline CLIENT: & Bechtel Nevada & CLIENT ID: & EMAD-VPOR-3 \\
PROJECT ID: & V553/17777 & DATE SAMPLED: & $12 / 1 / 98$ \\
PROJECT \#: & 17777 & NEL SAMPLE ID: $19812048-03$ \\
& & & \\
TEST: & TCLP by EPA 1311, July 1992 \& Volatile Organics by EPA 8260B, Dec. 1996 \\
METHOD: & EPA 8260A & TCLP EXTRACT DATE: & $12 / 9 / 98$ \\
MATRIX: & Solid & EXTRACTED: & $12 / 11 / 98$ \\
DILUTION: & 1 & ANALYZED: & $12 / 11 / 98$
\end{tabular}

\begin{tabular}{lcc}
\hline PARAMETER & $\begin{array}{c}\text { Result } \\
\mathbf{m g} / \mathrm{L}\end{array}$ & $\begin{array}{c}\text { Reporting } \\
\text { Limit }\end{array}$ \\
\cline { 2 - 3 } Benzene & $\mathrm{ND}$ & $0.1 \mathrm{mg} / \mathrm{L}$ \\
Carbon tetrachloride & $\mathrm{ND}$ & $0.1 \mathrm{mg} / \mathrm{L}$ \\
Chlorobenzene & $\mathrm{ND}$ & $0.1 \mathrm{mg} / \mathrm{L}$ \\
Chloroform & $\mathrm{ND}$ & $0.1 \mathrm{mg} / \mathrm{L}$ \\
1.t-Dichlorobenzene (p-DCB) & $\mathrm{ND}$ & $0.1 \mathrm{mg} / \mathrm{L}$ \\
1.2-Dichloroethane (1,2-DCA) & $\mathrm{ND}$ & $0.1 \mathrm{mg} / \mathrm{L}$ \\
l.1-Dichloroethene (1,1-DCE) & $\mathrm{ND}$ & $0.1 \mathrm{mg} / \mathrm{L}$ \\
Methyl Ethyl Ketone & $\mathrm{ND}$ & $2 \mathrm{mg} / \mathrm{L}$ \\
Tetrachloroethene (PCE) & $\mathrm{ND}$ & $0.1 \mathrm{mg} / \mathrm{L}$ \\
Trichioroethene & $\mathrm{ND}$ & $0.1 \mathrm{mg} / \mathrm{L}$ \\
Vinyl chloride & $\mathrm{ND}$ & $0.1 \mathrm{mg} / \mathrm{L}$
\end{tabular}

\section{JUALITY CONTROL DATA:}

\section{iurrogate}

t-Bromofluorobenzene

Jibromofluoromethane

Toluene-d8

\section{\% Recovery}

86

93

99
Acceptable Range

$74-121$

$80-120$

$81-117$

VD - Not Detected

his report shall not be reproduced except in full, without the written approval of the laboratory. 
NEL LABORATORIES

\begin{tabular}{llll}
\hline CLIENT: & Bechtel Nevada & CLIENT ID: & EMAD-VPOR-3 \\
PROJECT ID: & V553/17.777 & DATE SAMPLED: $12 / 1 / 98$ \\
PROJECT \#: & 17777 & NEL SAMPLE ID: & L9812048-03 \\
& & & \\
TEST: & TCLP by EPA 1311, July 1992 \& Semivolatile & Organics by EPA Method 8270C, Dec. 1996 \\
METHOD: & EPA 8270 & TCLP EXTRACT DATE: $12 / 8 / 98$ \\
MATRIX: & Solid & EXTRACTED: & $12 / 10 / 98$ \\
DILUTION: & 1 & ANALYZED: & $12 / 13 / 98$
\end{tabular}

\section{PARAMETER}

1,4-1)ichlorobenzene (p-DCB)

2.4-Dinitrotoluene (DNT)

Hexachlorobenzene

Hexachlorobutadiene

Hexachloroethane

2-Methylphenol

3.4-Methylphenol (isomeric pair)

Nitrobenzene

Pentachlorophenol

Pyridine

2.4.5-Trichlorophenol

2.4.6-Trichlorophenol

\begin{tabular}{cc}
$\begin{array}{c}\text { Result } \\
\text { mg/L }\end{array}$ & \\
\cline { 1 - 1 } ND & $\mathrm{Jm}$ \\
$\mathrm{ND}$ & \\
$\mathrm{ND}$ & \\
$\mathrm{ND}$ & $\mathrm{Jm}$ \\
$\mathrm{ND}$ & $\mathrm{Jm}$ \\
$\mathrm{ND}$ & \\
$\mathrm{ND}$ & \\
$\mathrm{ND}$ & $\mathrm{Jm}$ \\
$\mathrm{ND}$ & \\
$\mathrm{ND}$ & $\mathrm{Jm}$ \\
$\mathrm{ND}$ & \\
$\mathrm{ND}$ &
\end{tabular}

\begin{tabular}{c}
$\begin{array}{c}\text { Reporting } \\
\text { Limit }\end{array}$ \\
\hline $0.1 \mathrm{mg} / \mathrm{L}$ \\
$0.1 \mathrm{mg} / \mathrm{L}$ \\
$0.1 \mathrm{mg} / \mathrm{L}$ \\
$0.1 \mathrm{mg} / \mathrm{L}$ \\
$0.1 \mathrm{mg} / \mathrm{L}$ \\
$0.1 \mathrm{mg} / \mathrm{L}$ \\
$0.1 \mathrm{mg} / \mathrm{L}$ \\
$0.1 \mathrm{mg} / \mathrm{L}$ \\
$0.1 \mathrm{mg} / \mathrm{L}$ \\
$0.1 \mathrm{mg} / \mathrm{L}$ \\
$0.1 \mathrm{mg} / \mathrm{L}$ \\
$0.1 \mathrm{mg} / \mathrm{L}$
\end{tabular}

2UALITY CONTROL DATA:

\section{jurrogate}

$\therefore$ 4,6-Tribromophenol

2-Fluorobiphenyl

?-Fluorophenol

Vitrobenzene-ds

)-Terphenyl-d 14

?henol-d5

\section{\% Recovery}

\section{6}

43

11

17

- 66

13

\section{Acceptable Range}

$10-123$

$43-116$

$21-100$

$35-114$

$33-141$

10- 94

JD - Not Detected

his report shall not be reproduced except in full, without the written approval of the laboratory. 
NEL LABORATORIES

\begin{tabular}{llll}
\hline CLIENT: & Bechtel Nevada & CLIENT ID: & EMAD-VPOR-4 \\
PROJECT ID: & V553/17777 & DATE SAMPLED: & $12 / 1 / 98$ \\
PROJECT \#: & 17777 & NEL SAMPLE ID: & L9812048-04 \\
& & & \\
TEST: & TCLP by EPA 1311, July 1992 \& Volatile & Organics by EPA 8260B, Dec. 1996 \\
METHOD: & EPA 8260A & TCLP EXTRACT DATE: $12 / 9 / 98$ \\
MATRIX: & Solid & EXTRACTED: & $12 / 11 / 98$ \\
DILUTION: & 1 & ANALYZED: & $12 / 11 / 98$
\end{tabular}

\begin{tabular}{lcc}
\hline PARAMETER & $\begin{array}{c}\text { Result } \\
\mathbf{m g} / \mathrm{L}\end{array}$ & $\begin{array}{c}\text { Reporting } \\
\text { Limit }\end{array}$ \\
\cline { 2 - 3 } & $\mathrm{ND}$ & $0.1 \mathrm{mg} / \mathrm{L}$ \\
Benzene & $\mathrm{ND}$ & $0.1 \mathrm{mg} / \mathrm{h}$ \\
Carbon tetrachloride & $\mathrm{ND}$ & $0.1 \mathrm{mg} / \mathrm{L}$ \\
Chlorobenzene & $\mathrm{ND}$ & $0.1 \mathrm{mg} / \mathrm{L}$ \\
Chloroform & $\mathrm{ND}$ & $0.1 \mathrm{mg} / \mathrm{L}$ \\
1.4-Dichlorobenzene (p-DCB) & $\mathrm{ND}$ & $0.1 \mathrm{mg} / \mathrm{L}$ \\
1.2-Dichloroethane (1,2-DCA) & $\mathrm{ND}$ & $0.1 \mathrm{mg} / \mathrm{L}$ \\
I.1-Dichloroethene (1,I-DCE) & $\mathrm{ND}$ & $2 . \mathrm{mg} / \mathrm{L}$ \\
Methyl Ethyl Ketone & $\mathrm{ND}$ & $0.1 \mathrm{mg} / \mathrm{L}$ \\
Tetrachloroethene (PCE) & $\mathrm{ND}$ & $0.1 \mathrm{mg} / \mathrm{L}$ \\
Trichloroethene & $\mathrm{ND}$ & $0.1 \mathrm{mg} / \mathrm{L}$
\end{tabular}

2UALITY CONTROL DATA:

iurrogate

t-Bromofluorobenzene

Jibromofluoromethane

Toluene-d8
$\%$ Recoverv

88

95

99
Acceptable Range

$74-121$

$80-120$

$81-117$

VD - Not Detected

This report shall not be reproduced except in full, without the written approval of the laboratory. 


$\begin{array}{llll}\text { CLIENT: } & \text { Bechtel Nevada } & \text { CLIENT ID: } & \text { EMAD-VPOR-4 } \\ \text { PROJECT ID: } & \text { V553/17777 } & \text { DATE SAMPLED: } 12 / 1 / 98 \\ \text { PROJECT \#: } & 17777 & \text { NEL SAMPLE ID: } & \text { L9812048-04 } \\ & & & \\ \text { TEST: } & \text { TCLP by EPA 1311, July } 1992 \text { \& Semivolatile Organics by EPA Method 8270C, Dec. } 1996 \\ \text { METHOD: } & \text { EPA } 8270 & \text { TCLP EXTRACT DATE: } 12 / 8 / 98 \\ \text { MATRIX: } & \text { Solid } & \text { EXTRACTED: } & 12 / 10 / 98 \\ \text { DILUTION: } & 1 & \text { ANALYZED: } & 12 / 13 / 98\end{array}$

\section{PARAMETER}

1.4-Dichlorobenzene ( $p-D C B$ )

2.4-Dinitrotoluene (DNT)

Hexachlorobenzene

Hexachlorobutadiene

Hexachloroethane

2-Nethylphenol

3.4-Methylphenol (isomeric pair)

Vitrobenzene

Pentachlorophenol

Pyridine

2.4.5-Trichlorophenol

2.4.6-Trichlorophenol

\begin{tabular}{cc}
$\begin{array}{c}\text { Result } \\
\text { mg/L }\end{array}$ & $\begin{array}{c}\text { Reporting } \\
\text { Limit }\end{array}$ \\
\cline { 2 - 2 } ND & $0.1 \mathrm{mg} / \mathrm{L}$ \\
ND & $0.1 \mathrm{mg} / \mathrm{L}$ \\
ND & $0.1 \mathrm{mg} / \mathrm{L}$ \\
ND & $0.1 \mathrm{mg} / \mathrm{L}$ \\
ND & $0.1 \mathrm{mg} / \mathrm{L}$ \\
ND & $0.1 \mathrm{mg} / \mathrm{L}$ \\
ND & $0.1 \mathrm{mg} / \mathrm{L}$ \\
ND & $0.1 \mathrm{mg} / \mathrm{L}$ \\
ND & $0.1 \mathrm{mg} / \mathrm{L}$ \\
ND & $0.1 \mathrm{mg} / \mathrm{L}$ \\
ND & $0.1 \mathrm{mg} / \mathrm{L}$ \\
ND & $0.1 \mathrm{mg} / \mathrm{L}$
\end{tabular}

QUALITY CONTROL DATA:

\section{Surrogate}

2.4.6-Tribromophenol

2-Fluorobiphenyl

$\therefore$-Fluorophenol

Vitrobenzene-d5

o-Terphenyl-d 14

Phenol-d5

\section{\% Recoverv}

67

67

35

58

60

25

\section{Acceptable Range}

$10-123$

$43-116$

$21-100$

$35-114$

$33-141$

$10-94$

\section{iD - Not Detected}

rinis report shall not be reproduced except in full, without the written approval of the laboratory. 
NEL LABORATORIES

\begin{tabular}{|c|c|c|c|c|}
\hline $\begin{array}{l}\text { CLIENT: } \\
\text { PROJECT ID: } \\
\text { PROJECT \#: }\end{array}$ & $\begin{array}{l}\text { Bechtel Nevada } \\
\text { V553/17777 } \\
17777\end{array}$ & $\begin{array}{l}\text { CLIENT ID: } \\
\text { DATE SAMPLED: } \\
\text { NEL SAMPLE ID: }\end{array}$ & $\begin{array}{l}\text { TB-120198 } \\
12 / 1 / 98 \\
\text { L9812048-05 }\end{array}$ & \\
\hline $\begin{array}{l}\text { TEST: } \\
\text { METHOD: } \\
\text { MATRIX: } \\
\text { DILUTION: }\end{array}$ & $\begin{array}{l}\text { TCLP by EPA } 1 \\
\text { EPA } 8260 \mathrm{~B} \\
\text { Aqueous } \\
1\end{array}$ & $\begin{array}{l}\text { ganics by EPA } 8260 \mathrm{~B}, \\
\text { TCLP EXTRACT DA } \\
\text { EXTRACTED: } \\
\text { ANALYZED: }\end{array}$ & $\begin{array}{ll}\text { Dec. } & 1996 \\
\text { ATE: } & 12 / 11 / 98 \\
& 12 / 11 / 98 \\
12 / 11 / 98\end{array}$ & \\
\hline \multicolumn{2}{|l|}{ PARAMETER } & $\begin{array}{c}\text { Result } \\
\mathrm{mg} / \mathrm{L}\end{array}$ & & $\begin{array}{c}\text { Reporting } \\
\text { Limit }\end{array}$ \\
\hline \multicolumn{2}{|l|}{$\overline{\text { Benzene }}$} & ND & & $0.1 \mathrm{mg} / \mathrm{L}$ \\
\hline \multicolumn{2}{|c|}{ Carbon tetrachioride } & ND & & $0.1 \mathrm{mg} / \mathrm{L}$ \\
\hline \multicolumn{2}{|c|}{ Chlorobenzene } & $N D$ & & $0.1 \mathrm{mg} / \mathrm{L}$ \\
\hline \multicolumn{2}{|l|}{ Chioroform } & ND & & $0.1 \mathrm{mg} / \mathrm{L}$ \\
\hline \multicolumn{2}{|c|}{ 1.4-Dichlorobenzene ( $p-D C B)$} & ND & & $0.1 \mathrm{mg} / \mathrm{L}$ \\
\hline \multicolumn{2}{|c|}{ 1.2-Dichloroethane (1,2-DCA) } & ND & & $0.1 \mathrm{mg} / \mathrm{L}$ \\
\hline \multicolumn{2}{|c|}{ 1.1-Dichloroethene (1,1-DCE) } & ND & & $0.1 \mathrm{mg} / \mathrm{L}$ \\
\hline \multicolumn{2}{|c|}{ Methyl Ethyl Ketone } & ND & & 2. $\mathrm{mg} / \mathrm{L}$ \\
\hline \multicolumn{2}{|c|}{ Tetrachloroethene (PCE) } & ND & & $0.1 \mathrm{mg} / \mathrm{L}$ \\
\hline \multicolumn{2}{|l|}{ Trichloroethene } & ND & & $0.1 \mathrm{mg} / \mathrm{L}$ \\
\hline \multicolumn{2}{|l|}{ Vinyl chloride } & ND & & $0.1 \mathrm{mg} / \mathrm{L}$ \\
\hline
\end{tabular}

QUALITY CONTROL DATA:

Surrogate

4-Bromofluorobenzene

Dibromofluoromethane

Toluene-d8
$\%$ Recovery

86

95

99
Acceptable Range

$86-115$

$86-118$

$88-110$

VD - Not Detected

This report shall not be reproduced except in full, without the written approval of the laboratory. 


$\begin{array}{llll}\text { CLIENT: } & \text { Bechtel Nevada } & \text { CLIENT ID: } & \text { Method Blank } \\ \text { PROJECT ID: } & \text { Soil Testing Apex Industrial Use Zone/3745 } & \text { DATE SAMPLED: } & \text { NA } \\ \text { PROJECT \#: } & 37456-002-16 & \text { NEL SAMPLE ID: } & 121098-E 3-T C L P-B L K \\ & & & \\ \text { TEST: } & \text { TCLP by EPA 1311, July } 1992 \text { \& Semivolatile Organics by EPA Method 8270C, Dec. } 1996 \\ \text { METHOD: } & \text { EPA 8270 } & \text { TCLP EXTRACT DATE: } 12 / 8 / 98 \\ \text { MATRIX: } & \text { TCLP Extract } & \text { EXTRACTED: } & 12 / 10 / 98 \\ & & \text { ANALYZED: } & 12 / 13 / 98\end{array}$

\section{PARAMETER}

1.4-Dichlorobenzene ( $p$-DCB)

2.4-Dinitrotoluene (DNT)

Hexachiorobenzene

Hexachlorobutadiene

Hexachloroethane

2-Mlethylphenol

3.4-Methyiphenol (isomeric pair)

Nitrobenzene

Pentachlorophenol

Puridine

2.4.5-Trichlorophenol

2.4.6-Trichlorophenol

\begin{tabular}{c}
$\begin{array}{c}\text { Result } \\
\mathbf{m g} / \mathbf{L}\end{array}$ \\
\hline ND \\
ND \\
ND \\
ND \\
ND \\
ND \\
ND \\
ND \\
ND \\
ND \\
ND \\
ND
\end{tabular}

\section{Reporting}

Limit

$0.1 \mathrm{mg} / \mathrm{L}$

$0.1 \mathrm{mg} / \mathrm{L}$

$0.1 \mathrm{mg} / \mathrm{L}$.

$0.1 \mathrm{mg} / \mathrm{L}$

$0.1 \mathrm{mg} / \mathrm{L}$

$0.1 \mathrm{mg} / \mathrm{L}$

$0.1 \mathrm{mg} / \mathrm{L}$

$0.1 \mathrm{mg} / \mathrm{L}$

$0.1 \mathrm{mg} / \mathrm{L}$

$0.1 \mathrm{mg} / \mathrm{L}$

$0.1 \mathrm{mg} / \mathrm{L}$

$0.1 \mathrm{mg} / \mathrm{L}$

\section{QUALITY CONTROL DATA:}

\section{Surrogate}

2.4.6-Tribromophenol

--Fluorobipheny!

--Fluorophenol

Vitrobenzene-d5

-Terphenyl-d 44

shenol-d5

$\checkmark D$ - Not Detected
\% Recovery

$$
.67
$$

43

60

62

29
Acceptable Range

$10-123$

$43-116$

$21-100$

$35-114$

$33-141$

$10-94$

This report shall not be reproduced except in full, without the written approval of the laboratory. 
NEL LABORATORIES

\begin{tabular}{|c|c|c|c|c|}
\hline $\begin{array}{l}\text { CLIENT: } \\
\text { PROJECT ID: } \\
\text { PROJECT \#: }\end{array}$ & $\begin{array}{l}\text { Bechtel Nevada } \\
\text { Soil Testing Apex Industrial Use Zone/3745 } \\
37456-002-16\end{array}$ & $\begin{array}{l}\text { CLIENT ID: } \\
\text { DATE SAMPLED: } \\
\text { NEL SAMPLE ID: }\end{array}$ & $\begin{array}{l}\text { Method Blank } \\
\text { NA } \\
981211-\text { TCLP-BLK }\end{array}$ & \\
\hline $\begin{array}{l}\text { TEST: } \\
\text { METHOD: } \\
\text { MATRIX: }\end{array}$ & $\begin{array}{l}\text { TCLP by EPA } 1311, \text { July } 1992 \text { \& Volatile } \\
\text { EPA } 8260 A \\
\text { TCLP Extract }\end{array}$ & $\begin{array}{l}\text { ganics by EPA 8260B } \\
\text { TCLP EXTRACT D } \\
\text { EXTRACTED: } \\
\text { ANALYZED: }\end{array}$ & $\begin{array}{ll}\text { 3, Dec. } & 1996 \\
\text { ATE: } & 12 / 9 / 98 \\
& 12 / 11 / 98 \\
12 / 11 / 98\end{array}$ & \\
\hline \multicolumn{2}{|l|}{ PARAMETER } & $\begin{array}{c}\text { Result } \\
\mathrm{mg} / \mathrm{L}\end{array}$ & & $\begin{array}{c}\text { Reporting } \\
\text { Limit }\end{array}$ \\
\hline \multicolumn{2}{|l|}{ Benzene } & ND & & $0.1 \mathrm{mg} / \mathrm{L}$ \\
\hline \multicolumn{2}{|c|}{ Carbon tetrachloride } & ND & & $0.1 \mathrm{mg} / \mathrm{L}$ \\
\hline \multicolumn{2}{|l|}{ Chlorobenzene } & ND & & $0.1 \mathrm{mg} / \mathrm{L}$ \\
\hline \multicolumn{2}{|l|}{ Chloroform } & ND & & $0.1 \mathrm{mg} / \mathrm{L}$ \\
\hline \multicolumn{2}{|c|}{ 1.4-Dichlorobenzene ( $p-D C B)$} & ND & & $0.1 \mathrm{mg} / \mathrm{L}$ \\
\hline \multicolumn{2}{|c|}{ 1.2-Dichloroethane (1.2-DCA) } & ND & & $0.1 \mathrm{mg} / \mathrm{L}$ \\
\hline \multicolumn{2}{|c|}{ 1.1-Dichloroethene (1.1-DCE) } & ND & & $0.1 \mathrm{mg} / \mathrm{L}$ \\
\hline \multicolumn{2}{|c|}{ Methỵl Ethyl Ketone } & ND & & $2 \mathrm{mg} / \mathrm{L}$ \\
\hline \multicolumn{2}{|c|}{ Tetrachloroethene (PCE) } & ND & & $0.1 \mathrm{mg} / \mathrm{L}$ \\
\hline \multicolumn{2}{|l|}{ Trichloroethene } & ND & & $0.1 \mathrm{mg} / \mathrm{L}$ \\
\hline \multicolumn{2}{|l|}{ Vinyl chloride } & ND & & $0.1 \mathrm{mg} / \mathrm{L}$ \\
\hline
\end{tabular}

QUALITY CONTROL DATA:

Surrogate

4-Bromofluorobenzene

Jibromofluoromethane

Toluene-d8
\% Recovery

87

.91

100
Acceptable Range

$74-121$

$80-120$

$81-117$

ND - Not Detected

This report shall not be reproduced except in full. without the written approval of the laboratory. 


\begin{tabular}{|c|c|c|c|c|}
\hline $\begin{array}{l}\text { CLIENT: } \\
\text { PROJECT ID: } \\
\text { PROJECT \#: }\end{array}$ & $\begin{array}{l}\text { Bechtel Nevada } \\
\text { Soil Testing Apex Industrial Use Zone/3745 } \\
37456-002-16\end{array}$ & $\begin{array}{l}\text { CLIENT ID: } \\
\text { DATE SAMPLED: } \\
\text { NEL SAMPLE ID: }\end{array}$ & $\begin{array}{l}\text { Method Blank } \\
\text { NA } \\
981211 \text { ATCLP-BLK }\end{array}$ & \\
\hline $\begin{array}{l}\text { TEST: } \\
\text { METHOD: } \\
\text { MATRIX: }\end{array}$ & $\begin{array}{l}\text { TCLP by EPA } 1311, \text { July } 1992 \text { \& Volatile O } \\
\text { EPA } 8260 B \\
\text { TCLP Extract }\end{array}$ & $\begin{array}{l}\text { ganics by EPA } 8260 B, \\
\text { TCLP EXTRACT DA } \\
\text { EXTRACTED: } \\
\text { ANALYZED: }\end{array}$ & $\begin{array}{ll}3, \text { Dec. } 1996 \\
\text { ATE: } 12 / 11 / 98 \\
12 / 11 / 98 \\
12 / 11 / 98\end{array}$ & \\
\hline$\frac{\text { PARAMETER }}{\text { Benzene }}$ & . & $\begin{array}{c}\text { Result } \\
\mathrm{mg} / \mathrm{L}\end{array}$ & & $\begin{array}{c}\text { Reporting } \\
\text { Limit }\end{array}$ \\
\hline $\begin{array}{l}\text { Benzene } \\
\text { Carbon tetrachlori }\end{array}$ & & ND & & $0.1 \mathrm{mg} / \mathrm{L}$ \\
\hline Chlorobenzene & & ND & & $0.1 \mathrm{mg} / \mathrm{L}$ \\
\hline Chloroform & & $\begin{array}{l}\text { ND } \\
\text { ND }\end{array}$ & & $0.1 \mathrm{mg} / \mathrm{L}$ \\
\hline 1.4-Dichlorobenze & ne $(p-D C B)$ & ND & & $\begin{array}{l}0.1 \mathrm{mg} / \mathrm{L} \\
0.1 \mathrm{mg} / \mathrm{L}\end{array}$ \\
\hline 1.2-Dichloroethan & $e(1,2-D C A)$ & ND & & $\begin{array}{l}0.1 \mathrm{mg} / \mathrm{L} \\
0.1 \mathrm{mg} / \mathrm{L}\end{array}$ \\
\hline 1.1-Dichloroethen & $(1,1-D C E)$ & ND & . & $0.1 \mathrm{mg} / \mathrm{L}$ \\
\hline Methyl Ethyl Keto & & ND & & $2 \mathrm{mg} / \mathrm{L}$ \\
\hline Tetrachloroethene & (PCE) & ND & $\cdot$ & $0.1 \mathrm{mg} / \mathrm{L}$ \\
\hline $\begin{array}{l}\text { Trichloroethene } \\
\text { Vinyl chloride }\end{array}$ & & ND & & $0.1 \mathrm{mg} / \mathrm{L}$ \\
\hline Vinyl chloride & & ND & & $0.1 \mathrm{mg} / \mathrm{L}$ \\
\hline
\end{tabular}

QUALITY CONTROL DATA:

\section{Surrogate}

t-Bromofluorobenzene

Dibromofluoromethane

Toluene-d8
$\%$ Recovery

87

.91

100
Acceptable Range

$86-115$

$86-118$

$88-110$

ND - Not Detected

This report shall not be reproduced except in full, without the written approval of the laboratory. 
NEL LABORATORIES

CLIENT: Bechtel Nevada

PROJECT NAME: V553

PROJECT NUMBER: 17777

TEST:

Total Extractable Petroleum Hydrocarbons by EPA Method 8015M, December 1996

METHOD: EPA 8015M

MATRIX: Solid

ANALYST: SEJ

\begin{tabular}{|c|c|c|c|c|c|c|c|c|}
\hline $\begin{array}{l}\text { CLIENT } \\
\text { SAMPLE ID } \\
\end{array}$ & $\begin{array}{c}\text { SAMPLE } \\
\text { DATE }\end{array}$ & $\begin{array}{c}\text { NEL } \\
\text { SAMPLE ID } \\
\end{array}$ & $\begin{array}{c}\text { RESULT } \\
\mathrm{mg} / \mathrm{kg} \\
\end{array}$ & C.R. & $\begin{array}{c}\text { Reporting } \\
\text { Limit }\end{array}$ & $\begin{array}{l}\text { Surrogate } \\
\text { Recovery* } \\
\end{array}$ & EXTRACTED & ANALYZED \\
\hline EMAD-VPOR-1 & $12 / 1 / 98$ & L9812048-01 & 5200 & 0 & 2000. $\mathrm{mg} / \mathrm{kg}$ & $130 \%$ & $12 / 9 / 98$ & $12 / 14 / 98$ \\
\hline EMAD-VPOR-2 & $12 / 1 / 98$ & L9812048-02 & $1700 \mathrm{Jm}$ & 0 & 100. $\mathrm{mg} / \mathrm{kg}$ & $145 \%$ & $12 / 9 / 98$ & $12 / 11 / 98$ \\
\hline EMAD-VPOR-3 & $12 / 1 / 98$ & L9812048-03 & 4700 & O & 200. $\mathrm{mg} / \mathrm{kg}$ & $100 \%$ & $12 / 9 / 98$ & $12 / 15 / 98$ \\
\hline EMAD-VPOR-4 & $12 / 1 / 98$ & L9812048-04 & 3700 & O & 200. $\mathrm{mg} / \mathrm{kg}$ & $130 \%$ & $12 / 9 / 98$ & $12 / 11 / 98$ \\
\hline
\end{tabular}

\section{CR: Carbon Range}

O Oil Range Organics (C12 to $\mathrm{C} 32$ ).

Note: The reporting limit for Oil Range Organics in soil is $50 \mathrm{mg} / \mathrm{kg}$.

QUALITY CONTROL DATA (Total for Gas and Diesel Range):

\section{Sample ID}

Blank, 981209STP -BLK

LCS, 981209STP - LCS
Result Acceptable Range Surrogate Recoverv* Sample Number

$\begin{array}{llcccc}\mathrm{ND} & < & 10 . \mathrm{mg} / \mathrm{kg} & 78 \% & \mathrm{NA} \\ 61 & \% & 57.5-114 \% & 107 \% & \text { NA }\end{array}$

ND - Not Detected

* Surrogate used was Octacosane, acceptance limits 60-121\% for aqueous samples, 54-130\% for solid samples.

This report shall not be reproduced except in full, without the written approval of the laboratory. 


\begin{tabular}{ll}
\hline CLIENT: & Bechtel Nevada \\
PROJECT ID: & V553/17777 \\
PROJECT \#: & 17777 \\
TEST: & Organochlorine Pesticides by EPA 8081A, Dec. 1996 \\
MATRIX: & Solid \\
\hline
\end{tabular}

\begin{tabular}{|c|c|c|c|c|c|c|c|}
\hline PARAMETER & NEL Sample ID & $\frac{\text { Spike }}{\text { Amount }}$ & $\frac{\text { Spike }}{\text { Result }}$ & $\frac{\text { Percent }}{\text { Recovery }}$ & & $\frac{\text { Acceptable }}{\text { Range }}$ & $\underline{\text { RPD }}$ \\
\hline Aldrin & 981208PSTS-LCS & 33.3 & 32.6 & 98 & & $73-128$ & \\
\hline Aldrin & 981208PSTS-LCSD & 33.3 & 32.3 & 97 & & $73-128$ & 0.9 \\
\hline Aldrin & L9812048-02-MS & 33.3 & 32 & 96 & & $73-128$ & \\
\hline Aldrin & L9812048-02-MSD & 33.3 & 37 & 111 & & $73-128$ & 14.5 \\
\hline alpha-BHC & 981208PSTS-LCS & 33.3 & 33.3 & 100 & & $68-133$ & \\
\hline alpha-BHC & 981208PSTS-LCSD & 33.3 & 32 & 96 & & $68-133$ & 4. \\
\hline beta-BHC & 981208PSTS-LCS & 33.3 & 36 & 108 & & $71-136$ & \\
\hline beta-BHC & 981208PSTS-LCSD & 33.3 & 35.6 & 107 & & $71-136$ & 1.1 \\
\hline delta-BHC & 981208PSTS-LCS & 33.3 & 34 & 102 & & $69-133$ & \\
\hline delta-BHC & 981208PSTS-LCSD & 33.3 & 33 & 99 & & $69-133$ & 3. \\
\hline gamma-BHC (Lindane) & 981208PSTS-LCS & 33.3 & 32.3 & 97 & & $69-125$ & \\
\hline gamma-BHC (Lindane) & 981208PSTS-LCSD & 33.3 & 32 & 96 & & $69-125$ & 0.9 \\
\hline gamma-BHC (Lindane) & L9812048-02-MS & 33.3 & 29 & 87 & & $69-125$ & \\
\hline gamma-BHC (Lindane) & L9812048-02-MSD & 33.3 & 33.6 & 101 & & $69-125$ & 14.7 . \\
\hline Alpha-chlordane & 981208PSTS-LCS & 33.3 & 34.3 & 103 & & $63-120$ & \\
\hline Alpha-chlordane & 981208PSTS-LCSD & 33.3 & 30.3 & 91 & & $63-120$ & 12.4 \\
\hline Gamma-chlordane & 981208PSTS-LCS & 33.3 & .33 & 99 & & $68-126$ & \\
\hline Gamma-chlordane & 981208PSTS-LCSD & 33.3 & 33 & 99 & & $68-126$ & 0 . \\
\hline $4,4^{\prime}-\mathrm{DDD}$ & 981208PSTS-LCS & 33.3 & 38 & 114 & & $76-127$ & \\
\hline 4,4'-DDD & 981208PSTS-LCSD & 33.3 & 36.3 & 109 & & $76-127$ & 4.6 \\
\hline,$+ 4^{\prime}-\mathrm{DDE}$ & 981208PSTS-LCS & 33.3 & 50.6 & 152 & J & $62-143$ & \\
\hline $4.4^{\prime}-\mathrm{DDE}$ & 981208PSTS-LCSD & 33.3 & 37.3 & 112 & & $62-143$ & 30.3 \\
\hline $4,4^{\circ}-\mathrm{DDT}$ & 981208PSTS-LCS & 33.3 & 50.9 & 153 & J & $70-151$ & \\
\hline,$+ 4^{\circ}-\mathrm{DDT}$ & 981208PSTS-LCSD & 33.3 & 38.6 & 116 & & $70-151$ & 27.5 \\
\hline,$+ 4^{\circ}-D^{-1 T}$ & L9812048-02-MS & 33.3 & 11.7 & 35 & J! & $70-151$ & \\
\hline $4,4^{\circ}-\mathrm{DDT}$ & L9812048-02-MSD & 33.3 & 11.6 & 35 & Jl & $70-151$ & 1. \\
\hline Dieldrin & 981208PSTS-LCS & 33.3 & 38.6 & 116 & & $84-135$ & \\
\hline Dieldrin & 981208PSTS-LCSD & 33.3 & 37 & 111 & & $84-135$ & 4.2 \\
\hline Dieldrin & L9812048-02-MS & 33.3 & 36.3 & 109 & & $84-135$ & \\
\hline Dieldrin & L9812048-02-MSD & 33.3 & 43.3 & 130 & & $84-135$ & 17.6 \\
\hline Endosulfan I & 981208PSTS-LCS & 33.3 & 42.6 & 128 & & $57-161$ & \\
\hline Endosulfan I & 981208 PSTS-LCSD & 33.3 & 43.3 & 130 & & $57-161$ & 1.6 \\
\hline Endosulfan II & 981208PSTS-LCS & 33.3 & 38.6 & 116 & & $76-137$ & \\
\hline Endosulfan II & 981208 PSTS-LCSD & 33.3 & 30.6 & 92 & & $76-137$ & 23.1 \\
\hline Endosulfan sulfate & 981208PSTS-LCS & 33.3 & 39.6 & 119 & & $74-144$ & \\
\hline Endosulfan sulfate & 981208PSTS-LCSD & 33.3 & 33.6 & 101 & & $74-144$ & 16.4 \\
\hline Endrin & 981208PSTS-LCS & 33.3 & 38.6 & 116 & & $75-146$ & \\
\hline Endrin & 981208PSTS-LCSD & 33.3 & 37 & 111 & & $75-146$ & 4.2 \\
\hline
\end{tabular}

This report shall not be reproduced except in full, without the written approval of the laboratory. 


$\begin{array}{ll}\text { CLIENT: } & \text { Bechtel Nevada } \\ \text { PROJECT ID: } & \text { V553/1 } 7777 \\ \text { PROJECT \#: } & 17777 \\ \text { TEST: } & \text { Organochlorine Pesticides by EPA 8081A, Dec. } 1996 \\ \text { MATRIX: } & \text { Solid }\end{array}$

PARAMETER
Endrin
Endrin
Endrin aldehyde
Endrin aldehyde
Endrin ketone
Endrin ketone
Heptachlor
Heptachlor
Heptachlor
Heptachlor
Heptachlor epoxide
Heptachlor epoxide
Methoxychlor
Methoxychlor

\section{PARAMETER}

Endrin

Endrin aldehyde

Endrin ketone

Heptachlor

NEL Sample ID
L9812048-02-MS
L9812048-02-MSD
981208PSTS-LCS
981208PSTS-LCSD
981208PSTS-LCS
981208PSTS-LCSD
981208PSTS-LCS
981208PSTS-LCSD
L9812048-02-MS
L9812048-02-MSD
981208PSTS-LCS
981208PSTS-LCSD
981208PSTS-LCS
981208PSTS-LCSD

\begin{tabular}{|c|c|c|c|c|c|}
\hline $\begin{array}{l}\text { Spike } \\
\text { Amount }\end{array}$ & $\frac{\text { Spike }}{\text { Result }}$ & $\frac{\text { Percent }}{\text { Recovery }}$ & & $\frac{\text { Acceptable }}{\text { Range }}$ & RPD \\
\hline 33.3 & 21 & 63 & $\mathrm{Jl}$ & $75-146$ & \\
\hline 33.3 & 22 & 66 & $\mathrm{Jl}$ & $75-146$ & 4.7 \\
\hline 33.3 & 44 & 132 & & $71-173$ & \\
\hline 33.3 & 34.6 & 104 & & $71-173$ & 23.9 \\
\hline 33.3 & 37 & 111 & & $90-160$ & \\
\hline 33.3 & 31 & 93 & & $90-160$ & 17.6 \\
\hline 33.3 & 32 & 96 & & $70-134$ & \\
\hline 33.3 & 31.6 & 95 & & $70-134$ & 1.3 \\
\hline 33.3 & 17 & 51 & JI & $70-134$ & \\
\hline 33.3 & 18.6 & 56 & $\mathrm{Jl}$ & $70-134$ & 9. \\
\hline 33.3 & 32 & 96 & & $75-129$ & \\
\hline 33.3 & 32 & 96 & & $75-129$ & 0. \\
\hline 33.3 & 33.6 & $10]$ & & $55-173$ & \\
\hline 33.3 & 32.3 & 97 & & $55-173$ & 3.9 \\
\hline
\end{tabular}

VD - Not Detected

This report shall not be reproduced except in full, without the written approval of the laboratory. 


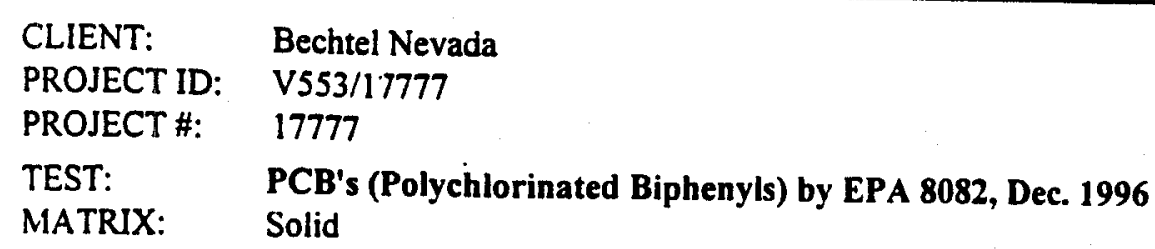

\section{PARAMETER}

Arochlor-1016

Arochlor-1016

Arochlor-1016

Arochlor-1016

Arochlor- 1260

Arochlor- 1260

Arochlor-1260

Arochlor- 1260

\section{NEL Sample ID}

981208PCBS-LCS

981208PCBS-LCSD

L9812048-02-MS

L9812048-02-MSD

981208PCBS-LCS

981208PCBS-LCSD

L9812048-02-MS

L9812048-02-MSD

\begin{tabular}{|c|c|c|c|c|}
\hline $\begin{array}{l}\text { Spike } \\
\text { Amount }\end{array}$ & $\begin{array}{l}\text { Spike } \\
\text { Result }\end{array}$ & $\underset{\text { Recovery }}{\text { Pecont }}$ & $\frac{\text { Acceptable }}{\text { Range }}$ & RPD \\
\hline 333 & 326 & 98 & $70-130$ & \\
\hline 333 & 289 & 87 & $70-130$ & 12 \\
\hline 333 & 354 & 106 & $70-130$ & \\
\hline 333 & 403 & 121 & $70-130$ & 4.1 \\
\hline 333 & 309 & 93 & $70-130$ & \\
\hline 333 & 261 & 78 & $70-130$ & 16.8 \\
\hline 333 & 317 & 95 & $48-129$ & \\
\hline 333 & 373 & 112 & $48-129$ & 16.2 \\
\hline
\end{tabular}




$\begin{array}{ll}\text { CLIENT: } & \text { Bechtel Nevada } \\ \text { PROJECT ID: } & \text { V553/17777 } \\ \text { PROJECT \#: } & 17777 \\ \text { TEST: } & \text { TCLP/STLC Metals } \\ \text { MATRIX: } & \text { Solid }\end{array}$

\begin{tabular}{|c|c|c|c|c|c|c|}
\hline PARAMETER & NEL Sample ID & $\underline{\text { Spike }}$ & $\begin{array}{l}\text { Spike } \\
\text { Result }\end{array}$ & $\frac{\text { Percent }}{\text { Recovery }}$ & $\frac{\text { Acceptable }}{\text { Range }}$ & RPD \\
\hline Arsenic & LI2048i-LCS & 0.5 & 0.495 & 99 & $85-115$ & \\
\hline Arsenic & L9812048-02-MS & 0.5 & 0.51 & 102 & $75-125$ & \\
\hline Arsenic & L9812048-02-MSD & 0.5 & 0.506 & 101 & $75-125$ & 0.8 \\
\hline Mercury & L12048-HG02-LCS & 0.005 & 0.00522 & 104 & $85-115$ & \\
\hline Mercury & L9812048-02-MS & 0.05 & 0.0559 & 112 & $75-125$ & \\
\hline Mercury & L9812048-02-MSD & 0.05 & 0.0566 & 113 & $75-125$ & 1.2 \\
\hline Silver & L12048i-LCS & 0.5 & 0.463 & 93 & $85-115$ & \\
\hline Silver & L9812048-02-MS & 0.5 & 0.466 & 93 & $75-125$ & \\
\hline Silver & L9812048-02-MSD & 0.5 & 0.472 & 94 & $75-125$ & 1.3 \\
\hline Barium & L12048i-LCS & 1 & 1.11 & $11 !$ & $85-115$ & \\
\hline Barium & L9812048-02-MS & 1 & 0.95 & 95 & $75-125$ & \\
\hline Barium & L9812048-02-MSD & 1 & 0.955 & 96 & $75-125$ & 0.5 \\
\hline Cadmium & L12048i-LCS & 0.2 & 0.188 & 94 & $85-115$ & \\
\hline Cadmium & L9812048-02-MS & 0.2 & 0.198 & 99 & $75-125$ & \\
\hline Cadmium & L9812048-02-MSD & 0.2 & 0.197 & 99 & $75-125$ & 0.5 \\
\hline Chromium & L12048i-LCS & 0.5 & 0.486 & 97 & $85-115$ & \\
\hline Chromium & L9812048-02-MS & 0.5 & 0.49 & 98 & $75-125$ & \\
\hline Chromium & L9812048-02-MSD & 0.5 & 0.494 & 99 & $75-125$ & 0.8 \\
\hline Lead & L12048i-LCS & 1 & 0.927 & 93 & $85-115$ & \\
\hline Lead & L9812048-02-MS & 1 & 1.05 & 105 & $75-125$ & \\
\hline Lead & L9812048-02-MSD & 1 & 1.01 & 101 & $75-125$ & 3.9 \\
\hline Selenium & L12048i-LCS & 0.5 & 0.526 & 105 & $85-115$ & \\
\hline Selenium & L9812048-02-MS & 0.5 & 0.576 & 115 & $75-125$ & \\
\hline Selenium & L9812048-02-MSD & 0.5 & 0.531 & 106 & $75-125$ & 8.1 \\
\hline
\end{tabular}

SD - Not Detected

This report shall not be reproduced except in full, without the written approval of the laboratory. 


\section{CLIENT: Bechtel Nevada}

PROJECT ID: V553/17777

PROJECT \#: $\quad 17777$

TEST: $\quad$ TCLP by EPA 1311, July 1992 \& Semivolatile Organics by EPA Method 8270C, Dec. 1996 MATRIX: Solid

\section{PARAMETER}

1,4-Dichlorobenzene ( $\mathrm{p}-\mathrm{DCB}$ )

1,4-Dichlorobenzene ( $\mathrm{p}-\mathrm{DCB})$

2.4-Dinitrotoluene (DNT)

2.4-Dinitrotoluene (DNT)

Pentachlorophenol

Pentachlorophenol

NEL Sample ID
121098-E3-TCLP-LCS
121098-E3-TCLP-LCSD
121098-E3-TCLP-LCS
121098-E3-TCLP-LCSD
121098-E3-TCLP-LCS
121098-E3-TCLP-LCSD

NEL Sample ID

121098-E3-TCLP-LCS

121098-ES-TCLPLCSD

121098-E3-TCLP-LCSD

121098-E3-TCLP-LCSD

\begin{tabular}{|c|c|c|c|c|}
\hline $\begin{array}{c}\text { Spike } \\
\text { Amount }\end{array}$ & $\frac{\text { Spike }}{\text { Result }}$ & $\frac{\text { Percent }}{\text { Recovery }}$ & $\frac{\text { Acceptable }}{\text { Range }}$ & RPD \\
\hline 80 & 51.5 & 64 & 36.97 & \\
\hline 80 & 54.2 & 68 & $36-97$ & 5.1 \\
\hline 80 & 56.4 & 71 & $24-96$ & $\omega$ \\
\hline 80 & 61.5 & 77 & $24-96$ & 8.7 \\
\hline 80 & 56.8 & 71 & $9-103$ & \\
\hline 80 & 62.5 & 78 & $9-103$ & 9.6 \\
\hline
\end{tabular}

VD - Not Detected

This report shall not be reproduced except in full, without the written approval of the laboratory. 


\section{NEL LABORATORIES}

CLIENT: Bechtel Nevada

PROJECT ID: V553/17777

PROJECT \#: $\quad 17777$

TEST: $\quad$ TCLP by EPA 1311, July 1992 \& Volatile Organies by EPA 8260B, Dec. 1996

MATRIX: Solid

\section{PARAMETER}

1,1-Dichloroethene (1,1-DCE)

1.1-Dichloroethene (1,1-DCE)

1,1-Dichloroethene (1,1-DCE)

Benzene

Benzene

Benzene

Chlorobenzene

Chlorobenzene

Chlorobenzene

Toluene

Toluene

Toluene

Trichloroethene (TCE)

Trichloroethene (TCE)

Trichloroethene (TCE)

NEL Sample ID
981211-TCLP-LCS
L9812048-02-MS
L9812048-02-MSD
981211-TCLP-LCS
L9812048-02-MS
L9812048-02-MSD
981211-TCLP-LCS
L9812048-02-MS
L9812048-02-MSD
981211-TCLP-LCS
L9812048-02-MS
L9812048-02-MSD
981211-TCLP-LCS
L9812048-02-MS
L9812048-02-MSD

NEL Sample 1D

L9812048-02-MS

L9812048-02-MS

L9812048-02-MSD

$\begin{array}{cccc}\frac{\text { Spike }}{\text { Amount }} & & \frac{\text { Spike }}{\text { Result }} & \frac{\text { Percent }}{\text { Recovery }} \\ 50 & 54.09 & 108 \\ 50 & 41 & 82 \\ 50 & 43 & 86 \\ 50 & 45.9 & 92 \\ 50 & 40 & 80 \\ 50 & 39 & 78 \\ 50 & 49.75 & 100 \\ 50 & 44 & 88 \\ 50 & 42 & 84 \\ 50 & 45 & 90 \\ 50 & 33 & 66 \\ 50 & 34 & 68 & \mathrm{Jl} \\ 50 & 42.8 & 86 \\ 50 & 38 & 76 \\ 50 & 37 & 74\end{array}$

Acceptable

Range RPD

$70-130$

$61-145$

$61-145$

4.8

$70-130$

$76-127$

$76-127$

$70-130$

$75-130$

$75 \cdot 130$

$70-130$

$76-125$

$76-125$

$70-130$

$71-120$

$71-120$

JD - Not Detected

This report shall not be reproduced except in full, without the written approval of the laboratory. 


\section{Bechtel Nevada Corporation}

ANALYTICAL SERVICES LABORATORY

P.O.Box 3936, N. Las Vegas, NV 89036

Reported to: Environmental Restoration

CAU 297 EMAD

J, F. Bonn, M/S NTS306

Report Date: 8-DEC-s

Sample Delivery Group: $B 979$

Batch: 0861

Report No. :

Program: 720

\begin{tabular}{|c|c|c|c|c|c|c|c|c|c|c|c|c|c|c|c|c|}
\hline $\begin{array}{c}\text { Sample } \\
\text {..... } \\
\text { Identification ..... }\end{array}$ & Isotope & Analysis & Result & $\begin{array}{c}\text { Error } \\
\%\end{array}$ & $\begin{array}{l}\text { Qual } \\
\text { Flag }\end{array}$ & $M D C$ & \begin{tabular}{|l|} 
Result \\
Units
\end{tabular} & $\begin{array}{c}\text { Analysis } \\
\text { Date }\end{array}$ & $\begin{array}{l}\text { Sample } \\
\text { Coll Date }\end{array}$ & Size & $\begin{array}{l}\text { Size } \\
\text { Units }\end{array}$ & \begin{tabular}{|l|} 
Type \\
Matrix \\
\end{tabular} & \begin{tabular}{|l|} 
Tracer \\
Yiold \%
\end{tabular} & \begin{tabular}{|l|} 
Spike \\
Recv \%
\end{tabular} & \begin{tabular}{|l|} 
System \\
Detector
\end{tabular} & $\begin{array}{l}\text { Packet-Item } \\
\text { Sample }\end{array}$ \\
\hline EMAD-VPOR-3 & $\operatorname{CS} 137$ & GAM 20 & $1.65 E-01$ & $6.1 E+01$ & & $5.8 E-02$ & pCi/g & $12-02.98$ & $12.01-98$ & $5.15 E+02$ & $g m$ & soll G & & & 05.01 & Ho177-0.7 \\
\hline EMAD-VPOR-3 & K 40 & GAM20 & $2.49 E+01$ & $2.0 E+01$ & & 7.0E-01 & pCi/g & 12-02-98 & 12.01 .98 & $5.15 E+02$ & $\mathrm{gm}$ & soll G & & & 05-01 & Ho177-O \\
\hline EMAD-VPOR-3 & RA226 & GAM20 & $1.20 E+\infty 0$ & $2.8 E+01$ & & $1.7 E-01$ & pCi/g & 12-02-98 & $12-01-98$ & $5.15 E+02$ & $\mathrm{gm}$ & soll G & & & 05.01 & Ho177-0.75 \\
\hline EMAD-VPOR-3 & TH228 & GAM20 & $2.28 E+00$ & $1.9 E+01$ & & $1.6 E-01$ & $\mathrm{pCi} / \mathrm{g}$ & $12-02-98$ & $12.01-98$ & $5.15 E+02$ & $\mathrm{gm}$ & soll G & & & 05.01 & Ho177.0.7 \\
\hline EMAD-VPOR-3 & TH232 & GAM20 & $2.41 E+00$ & $3.1 E+01$ & & 3.3E-01 & $p C i / g$ & $12-02.98$ & $12.01-98$ & $5.15 E+02$ & $\mathrm{gm}$ & SOIL G & & & 05.01 & Ho177-O \\
\hline EMAD-VPOR-4 & K 40 & GAM20 & $1.96 E+01$ & $2.1 E+01$ & & $6.5 E-01$ & pCi/g & $12-02-98$ & $12-01-98$ & $7.52 E+02$ & $g m$ & IL G & & & 05.01 & Ho177-1.75 \\
\hline EMAD-VPOR-4 & RA226 & GAM20 & $1.01 E+00$ & $3.0 E+01$ & & $1.5 E-01$ & $\mathrm{pCi} / \mathrm{g}$ & $12-02-98$ & $12-01-98$ & $7.52 \mathrm{E}+02$ & $g m$ & SOIL G & & & $05-01$ & H0177-1.7527 \\
\hline EMAD-VPOR-4 & TH228 & GAM20 & $1.84 E+00$ & $2.0 E+01$ & & $1.5 E-01$ & pCilg & $12.02-98$ & $12-01-98$ & $7.52 E+02$ & $\mathrm{gm}$ & soll G & & & 05.01 & HO177-1.7527 \\
\hline EMAD-VPOR-4 & TH232 & GAM20 & $1.26 E+00$ & $4.1 E+01$ & & 3.0E-01 & pCilg & 12.02-98 & $12-01-98$ & $7.52 E+02$ & $g m$ & SOIL G & & & 05.01 & HO177-1-7527 \\
\hline OA BKG EMPTY BTL U05 & No Nucl Det & GAM20 & $0.00 E+00$ & $0.0 E+00$ & $u$ & $0.0 E+00$ & NA & $12.02-98$ & 07.01-98 & $1.00 E+00$ & $\mathrm{gm}$ & QUAL & & & 05.01 & $0.0631-0.00$ \\
\hline
\end{tabular}

Comment:

Data generated from 20 minute gamma spectroscopy of samples submitted to the laboratory on 12/2/98.

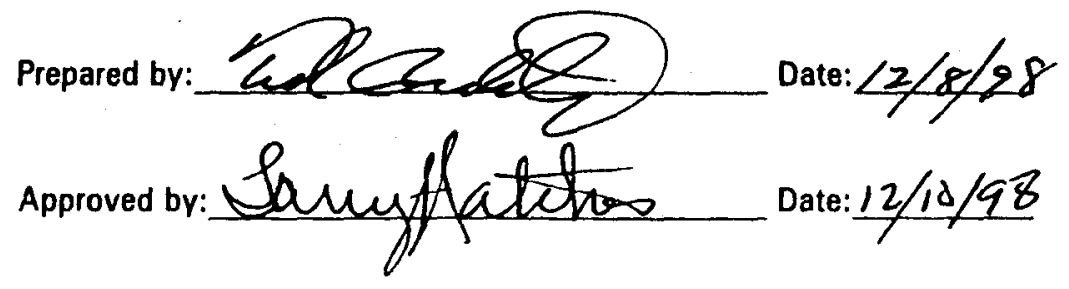

Qualification Flags:

$E=$ Estimated Quantity

$H=$ High Recovery for Sample

$\mathrm{J}=$ Result is less than the RDL

$L=$ Low Recovery for Sample

$P=$ Preliminary Results

O = Bad Instrument Quality Control, Result is OK

$R=$ Results are Unusable, Resampling is Necessary

$U=$ Result is less than Minimum Detectable Activity

Note: \% Error is the 2.0 Sigma $\mathrm{Er}$ 


\section{Bechtel Nevada Corporation}

ANALYTICAL SERVICES LABORATORY

P.O.Box 3936, N. Las Vegas, NV 89036

Reported to: Environmental Restoration

CAU 297 EMAD

J. F. Bonn, M/S NTS306

Report Date: 8-DEC Sample Delivery Group: B979.

Batch: 0861

Program: 500

Report No. :

\begin{tabular}{|c|c|c|c|c|c|c|c|c|c|c|c|c|c|c|c|c|}
\hline $\begin{array}{c}\text { Sample } \\
\ldots . . .\end{array}$ & Isotope & Analysis & Result & $\begin{array}{c}\text { Error } \\
\%\end{array}$ & $\begin{array}{l}\text { Qual } \\
\text { Flag }\end{array}$ & MDA & $\begin{array}{l}\text { Result } \\
\text { Units }\end{array}$ & $\begin{array}{c}\text { Analysis } \\
\text { Date }\end{array}$ & $\begin{array}{l}\text { Sample } \\
\text { Coll Date }\end{array}$ & Size & \begin{tabular}{|l|} 
Size \\
Units
\end{tabular} & \begin{tabular}{|c|} 
Type \\
Matrix
\end{tabular} & $\begin{array}{l}\text { Tracer } \\
\text { Yield \% }\end{array}$ & \begin{tabular}{|l|} 
Spike \\
Recv \%
\end{tabular} & $\begin{array}{l}\text { System } \\
\text { Detector }\end{array}$ & $\begin{array}{l}\text { Packet-Item } \\
\text { Samplo }\end{array}$ \\
\hline QA BKG EMPTY BTL UO5 & AM241 & GAM20 & $0.00 E+00$ & $0.0 \mathrm{E}+00$ & $u$ & $2.0 E+01$ & $\mathrm{pCi}$ & $12-02-98$ & $07-01-98$ & $1.00 E+00$ & $\mathrm{sm}$ & QUAL & & & 05.01 & $00631-0$. \\
\hline QA BKG EMPTY BTL U05 & C060 & GAM20 & $0.00 E+00$ & $0.0 E+00$ & u & $1.3 E+01$ & $\mathrm{pCi}$ & 12.02 .98 & $07.01-98$ & $1.00 E+\infty$ & $s m$ & QUAL & & & 05.01 & 00631 \\
\hline QA BKG EMPTY BTL UO5 & CS137 & GAM20 & $0.00 E+00$ & $0.0 E+00$ & u & $6.2 E+\infty$ & $\mathrm{pCi}$ & $12-02-98$ & $07-01-98$ & $1.00 E+00$ & $\mathbf{s m}$ & QUAL & & & $05-01$ & 00631.0 .0 \\
\hline OA SP:NAS-A0271 & AM241 & GAM20 & $1.80 E+05$ & $8.8 E+00$ & & $2.7 E+02$ & $\mathrm{pCi}$ & $12-02-98$ & $04-01-92$ & $1.00 E+\infty \mid$ & $\mathrm{sm}$ & QUAL & & 110.8 & $05-01$ & $00630-3$ \\
\hline QA SP:NAS-A0271 & C060 & GAM20 & $2.96 E+05$ & $8.6 E+00$ & & $2.1 E+02$ & $\mathrm{pCi}$ & 12-02-98 & $04-01-92$ & $1.00 E+\infty$ & $\mathbf{s m}$ & OUAL & & 99.4 & 05.01 & $00630-3$. \\
\hline QA SP:NAS-A0271 & CS137 & GAM20 & $2.04 E+05$ & $8.1 E+\infty 0$ & & $5.8 E+01$ & $\mathrm{pCi}$ & 12-02-98 & 04-01-92 & $|1.00 E+\infty|$ & $\mathbf{s m}$ & aUAL & & 100.6 & 05.01 & 00630-3- \\
\hline
\end{tabular}

Comment:

Data generated from 20 minute gamma spectroscopy of samples

submitted to the laboratory on $12 / 2 / 98$.

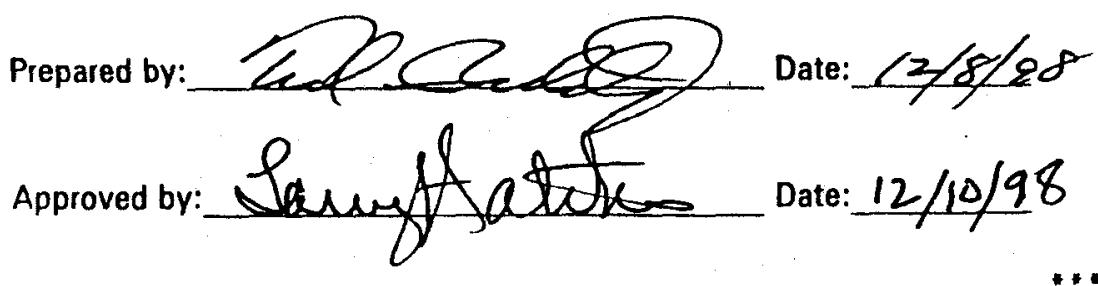

Qualification Flags:

$\mathbf{E}=$ Estimated Quantity

$H=$ High Recovery for Sample

$\mathrm{J}=$ Result is less than the RDL

$\mathrm{L}=$ Low Recovery for Sample

$\mathbf{P}=$ Preliminary Results

Q = Bad Instrument Quality Control, Result is OK

$\mathrm{R}=$ Results are Unusable, Resampling is Necessary

$\mathrm{U}=$ Result is less than Minimum Detectable Activity
Note: \% Error is the 2.0 Sigma

**** End af Rennrt **** 
$C^{-} \cdot T-F=3$

\section{Bechtel Nevada}

PROJECT/CLIENT INFORMATION

\begin{tabular}{|c|c|c|c|c|c|}
\hline \multicolumn{3}{|c|}{ PROJECT/ CLIENT INFORMATION } & \multicolumn{3}{|c|}{ REPORT INFORMATION } \\
\hline Project CAU 297 EMAD & VPOES & BN Org:: 2152 & \multicolumn{3}{|c|}{ Send Report lo: JERRT BUNN } \\
\hline \multicolumn{2}{|l|}{ Charge No: CTOIFO 3} & ASL Prog.: & Phone: $5-7341$ & Fax: $5 .-7761$ & MV: $N$ T5306 \\
\hline \multicolumn{3}{|c|}{ Project Manager: JeRpY bCiNN } & \multicolumn{3}{|c|}{ 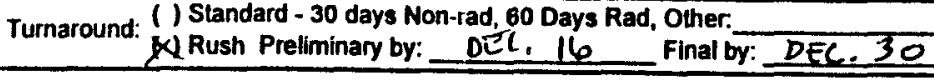 } \\
\hline Phone: -7341 & $5-77$ & M/S: 306 & Final report format: () St & ard XNTS-WAC (1)O & \\
\hline
\end{tabular}

ANALYTICAL SERVICES LABORATORY

SERVICES REQUEST \& CHAIN OF CUSTODY RECORD

Page

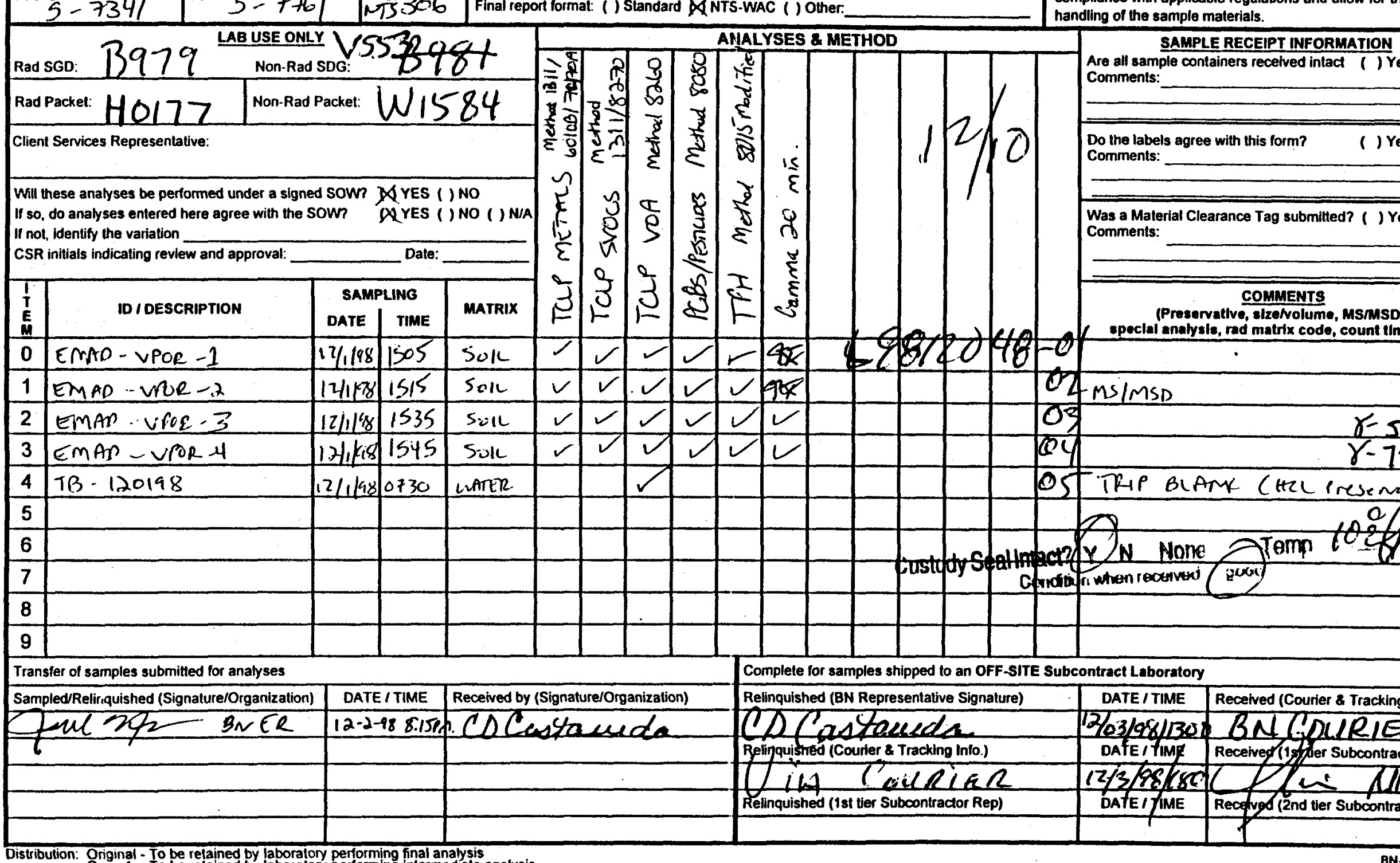

Distribution: Original - To be relained by laboratory performing final anahysis

Copy 2 : Ho be reained by Anaytical Services Laboralory
Copy 3 To be retained by sampler

Sampling Site: EMAP VACUMA The samples submitted contain (check) ()) Hazardous () Radioactlve

\uni Attach a brief narrative sumn Jentifying contaminants. This information will ensure compliance with applicable regulations and allow for th (nding of the sample malerials.

SAMPLE RECEIPT INFORMATION Are all sample containers received intact $(T) Y$

(1)Ye

Nas a Materil Ciearanco Tag submitted? ( ) Y Comments:

If not, identify the variation

Complete for samples shipped to an OFF-SITE Subcontract Laboratory 


\section{DISTRIBUTION LIST}




\section{DISTRIBUTION LIST}

\section{Nevada Department of Environmental Protection}

Paul Liebendorfer

2 (Controlled)*

Bureau of Federal Facilities

Division of Environmental Protection

333 W. Nye Lane, Room 13B

Carson City, NV 89706-0866

Michael McKinnon, Las Vegas Office

Bureau of Federal Facilities

1 (Controlled)*

Division of Environmental Protection

555 E. Washington, Suite 4300

Las Vegas, NV 89010

\section{U.S. Department of Energy}

Janet Appenzeller-Wing

1 (Uncontrolled)*

Environmental Restoration Division

U.S. Department of Energy, Nevada Operations Office

P.O. Box $98518 \mathrm{M} / \mathrm{S} 505$

Las Vegas, NV 89193-8518

Clayton Barrow

1 (Uncontrolled)*

Environmental Restoration Division

U.S. Department of Energy, Nevada Operations Office

P.O. Box $98518 \mathrm{M} / \mathrm{S} 505$

Las Vegas, NV 89193-8518

Sabrina Lawrence

Environmental Restoration Division

1 (Controlled)*

U.S. Department of Energy, Nevada Operations Office

P.O. Box $98518 \mathrm{M} / \mathrm{S} 505$

Las Vegas, NV 89193-8518

DOE Public Reading Facility

P.O. Box $98521 \mathrm{M} / \mathrm{S}$ NLV040

1 (Controlled)

Las Vegas, NV 89193-8521

DOE/Nevada Operations Office

1 (Uncontrolled)

Technical Information Resource Center

P.O. Box $98521 \mathrm{M} / \mathrm{S} 505$

Las Vegas, NV 89193-8521 


\section{DISTRIBUTION LIST (Continued)}

U.S. Department of Energy

2 (Uncontrolled)

Office of Scientific and Technical Information

175 Oak Ridge Turnpike

P.O. Box 62

Oak Ridge, TN 37831

\section{Bechtel Nevada}

Correspondence Control

1 (Uncontrolled)*

Bechtel Nevada

P.O. Box $98521 \mathrm{M} / \mathrm{S}$ NLV008

Las Vegas, NV 89193-8521

Jerry Bonn

1 (Uncontrolled)*

Bechtel Nevada

P.O. Box $98521 \mathrm{M} / \mathrm{S}$ NTS306

Las Vegas, NV 89193-8521

David Cowser

1 (Uncontrolled)*

Bechtel Nevada

P.O. Box $98521 \mathrm{M} / \mathrm{S}$ NLV082

Las Vegas, NV 89193-8521

Ann Heidema

1 (Uncontrolled)

Bechtel Nevada

P.O. Box $98521 \mathrm{M} / \mathrm{S}$ NLV022

Las Vegas, NV 89193-8521

Steve Nacht

1 (Uncontrolled)*

Bechtel Nevada

P.O. Box $98521 \mathrm{M} / \mathrm{S}$ NTS306

Las Vegas, NV 89193-8521

Jerel Nelson

1 (Uncontrolled)*

Bechtel Nevada

P.O. Box $98521 \mathrm{M} / \mathrm{S}$ NTS306

Las Vegas, NV 89193-8521 